\title{
Emergency Medicine Society of South Africa guidelines for the training and credentialing in emergency point-of-care ultrasound
}

\author{
M Wells, MB BCh, PhD, FCEM (SA); L N Goldstein, MB BCh, MD, FCEM (SA), Cert Crit Care (SA), \\ C Beringer, MB BCh, MMed, FCEM (SA)
}

Division of Emergency Medicine, Department of Family Medicine, Faculty of Health Sciences, University of the Witwatersrand, Johannesburg, South Africa

Corresponding author: M Wells (profmikewells@gmail.com)

S Afr Med J 2021;111(5):491-512. https://doi.org/10.7196/SAMJ.2021.v111i5.15346

\section{Executive summary Background}

The Emergency Medicine Society of South Africa (EMSSA) has provided training and credentialing in emergency point-of-care ultrasound (ePoCUS) since 2008. Since the use of ePoCUS has changed in the last decade and educational methods have evolved, it has become necessary to update and modify our guidelines, which are reported here. This document contains the full and detailed updated guidelines in five parts:

Part 1: EMSSA emergency point-of-care ultrasound training.

Part 2: Credentialing in emergency point-of-care ultrasound.

Part 3: Curriculum for core emergency point-of-care ultrasound training.

Part 4: Curriculum for advanced emergency point-of-care ultrasound training.

Part 5: Quality management in emergency point-of-care ultrasound.

The executive summary will highlight the important changes from the previous guidelines. The rationale for these changes will be expounded in the individual sections of the document.

The main difference between this ePoCUS guideline and other international policies is that there is a greater focus on credentialing in the South African (SA) guidelines while international guidelines focus more on the details of ePoCUS training. As patient safety is paramount, we believe that competency is crucial, and the outcomes of specific training focuses can be variable. We acknowledge the lack of good-quality evidence regarding specific training pathways or mechanisms that are required to produce a competent ePoCUS user. While we have made recommendations based on the best available evidence, this evidence does not take individual users' differences into account. Since competency is the endpoint, we have focused on a process that will ensure that we evaluate candidates comprehensively to establish this competency. It is necessary to concede that there is limited evidence to show that any competency examination accurately predicts safe and effective ePoCUS users in real-world circumstances. Nonetheless, we feel that this is the best interpretation of the current evidence and the most effective system currently available.

Three key concepts were considered in the formulation of these guidelines:

1. What types of ePoCUS scans are needed by clinicians practising in the Emergency Department (ED) based on the types of emergency patient presentations that are most prevalent and/or have the greatest threat to life?
2. What types of scans are needed in the training process from an educational perspective to allow for the development of appropriate ultrasound skills?

3. What is the most appropriate evidence-based method of credentialing users?

\section{Process}

Current published guidelines for ePoCUS from international organisations were reviewed in the creation of this document. ${ }^{[1-6]}$ In addition, publications related to ePoCUS training, credentialing and the clinical needs within EDs were identified. These guidelines and publications were then evaluated for strength of evidence and consensus was obtained regarding which processes and applications were most appropriate in the SA context. This consensus took place over several meetings in 2018 and 2019 between local ultrasound experts and EMSSA representatives. The resultant guidelines developed by EMSSA were then reviewed and approved by local SA experts in ePoCUS.

\section{Guidelines}

\section{Part 1: Changes from the previous position statement}

In this section, the changes and the rationale behind the changes of the previous position statement of 2009 are described. The major changes can be summarised as follows:

- Two distinct curricula have now been developed and the nomenclature for the courses has been changed to core ePoCUS and advanced ePoCUS.

- The modules included in the core ePoCUS curriculum have been changed.

- The training process has been changed, with the theoretical material offered as an online course.

- The advanced ePoCUS curriculum has been defined, in addition to a credentialing process for this curriculum.

- For both the core and advanced qualifications, the criteria to qualify for entrance to the credentialing process have been made easier and simpler. The credentialing assessment itself has been made more rigorous in terms of the scope and nature of the examination.

- A requirement for re-credentialing has been established to allow for better clinical governance and continuous audit of active ePoCUS providers.

- A more rigorous and transparent process of becoming an instructor and maintaining instructorship has been defined. 
- A policy on 'non-EMSSA' ePoCUS courses has been added. It includes the criteria for eligibility of participants from these courses to attend the EMSSA credentialing exam.

\section{Part 2: Key components of the credentialing process}

The following aspects of the credentialing process are covered in Part 2:

- Eligibility criteria for registering for the credentialing examination: any practitioner whose scope of practice includes ePOCUS may attempt the examination. There is no specific limitation on the type of course attended and the time elapsed since the course was done or the number of scans performed before the exam may be attempted.

- Components of the credentialing process: the first part of the examination will consist of a comprehensive computer-based theoretical multiple-choice questions (MCQs) and visual-aid questions (VAQs) examination. Thereafter, candidates will attempt a practical examination with simulated patients. The practical examination will focus on simulation of realistic scenarios to ensure the appropriate use, image generation and interpretation of ePoCUS over a range of clinical conditions.

- Assessment of the outcome of the examination: criteria for successful credentialing are detailed.

- Advanced curriculum modules will be credentialed individually.

- The requirements to maintain credentialed status: a 4-yearly re-credentialing assessment will be required for all ePoCUS users to maintain registration, including course coordinators and instructors.

\section{Part 3: Key components of the core curriculum}

The EMSSA 2020 guideline for the core ePoCUS curriculum now includes six core modules:

- Image acquisition and optimisation.

- Extended focused assessment with sonography in trauma (eFAST).

- Focused abdominal aorta ultrasound.

- Basic cardiac ultrasound.

- Basic lung ultrasound.

- Ultrasound-guided vascular access.

The changes from the 2009 guideline to the 2020 guideline include the following:

- Ultrasound for deep-vein thrombosis (DVT) assessment was removed as a core module because of new evidence suggesting that skills beyond basic ultrasound are required to perform this scan. In addition, this scan has been changed from a limited compression ultrasound protocol to an extended compression ultrasound protocol.

- The number of logged scans for each module was changed to a recommended number rather than a required number.

- There is a new course format: an online theory component and a hands-on practical session.

- The competency or credentialing assessment can be attempted at any stage rather than after a specified number of logged scans.

\section{Part 4: Key components of the advanced curriculum}

The EMSSA 2020 guideline for the advanced ePoCUS curriculum includes three mandatory core modules and two additional modules of the candidate's choice:
- Mandatory modules include:

- Focused cardiac ultrasound and haemodynamic assessment.

- DVT extended compression ultrasound.

- Advanced thoracic (lung) and airway ultrasound.

- Choice of optional modules (two to be selected):

- Regional anaesthesia and nerve blocks.

- Hepatobiliary and genito-urinary tract PoCUS.

- Gastrointestinal tract PoCUS.

Focused obstetric and gynaecological PoCUS.

Transcranial Doppler and ocular ultrasound.

The advanced ePoCUS course will use a similar flipped classroom style to the core ePoCUS course, with an online theoretical component followed by hands-on practical training for each module. EMSSA will assign a national clinical lead for each module (reviewed periodically), who will be responsible for training and credentialing of that module. Credentialing will be self-triggered by the candidate once they believe that they have achieved competency in a module. Each module will be assessed and credentialed separately. Once the candidate has completed the five modules, they will have completed the credentialing process. Advanced ePoCUS users will be regarded as competent in self-learning for additional ePoCUS applications, having proven advanced knowledge and skill, but will still have to re-credential every 4 years.

\section{Part 5: Quality management in emergency point-of-care ultrasound}

A quality management programme should be in place in every ED in which ePoCUS is used. Part 5 of this document provides a guideline as to what should be included in ePoCUS programmes: details of leadership, supervision of training, record-keeping, quality assurance with quality improvement and the management of ultrasound machines.

\section{Conclusions}

These guidelines are intended to assist trainers, training organisations and end-users in the processes of becoming an EMSSA-registered and credentialed ePoCUS user.

It is our hope that other specialist disciplines develop a similar guideline and process to ensure that a high standard of practice of point-of-care (PoCUS) in other environments is verifiably achieved.

The use or duplication of this policy will be permitted and encouraged upon request from EMSSA.

Acknowledgements. The authors would like to thank and acknowledge Dr Melanie Stander for her valued input in this position statement as well as all members (Dr David Cloete, Dr Elaine Erasmus, Dr Sian Geraty, Dr Jedd Hart, Dr Vidya Lalloo, Dr Pravani Moodley, Dr Pranesh Ramraj, Dr Dawie Schoeman, Dr Matthew Zoghby and Ms Mande Toubkin) of the EMSSA PoCUS SIG for reviewing, editing and approving the final document.

\footnotetext{
1. Society for Point-of-Care Ultrasound (SCOPUS). Guidelines for point-of-care ultrasound utilization in clinical practice. San Antonio: SCOPUS, 2018. https://spocus.org/wp-content/uploads/2019/12/ GUIDELINES-FOR-POINT-OF-CARE-ULTRASOUND-UTILISATION-IN-CLINICALPRACTICE.pdf (accessed 1 October 2020).

2. American Academy of Family Physicians (AAFP). Recommended curriculum guidelines for family medicine residents. Point-of-care ultrasound. Leawood: AAFP, 2018. https://www.aafp.org/dam/ AAFP/documents/medical_education_residency/program_directors/Reprint290D_POCUS.pdf (accessed 1 October 2020).

3. American College of Emergency Physicians (ACEP). Ultrasound guidelines: Emergency, pointof-care, and clinical ultrasound guidelines in medicine. Irving: ACEP, 2016. https://www.acep. org/patient-care/policy-statements/ultrasound-guidelines-emergency-point-of-care-and-clinicalultrasound-guidelines-in-medicine/ (accessed 1 October 2020)
} 
4. International Federation for Emergency Medicine (IFEM). Point-of-care ultrasound curriculum guidelines. Melbourne: IFEM, 2016. https://www.ifem.cc/wp-content/uploads/2016/07/IFEM-Pointguidelines. Melbourne: IFEM, 2016. https://www.ifem.cc/wp-content/uploads/20
of-Care-Ultrasound-Curriculum-Guidelines-2014.pdf (accessed 1 October 2020).

5. Society of Hosp Society of Hospital Medicine (SHM). Point-of-care ultrasound for hospitalists: A position stateme of the Society of Hospital Medicine. Philadelphia: SHM, 2019. https://mdedge-files-live.s3.us-east-2. amazonaws.com/files/s3fs-public/issues/articles/soni02780102e.pdf (accessed 1 October 2020).

6. Canadian Association of Emergency Physicians (CAEP). Recommendations for the use of pointof-care ultrasound (PoCUS) by emergency physicians in Canada. Ottawa: CAEP, 2018. https://caep. ca/wp-content/uploads/2018/11/CAEP-PoCUS-Position-Statement-Full-Text-2018-V7-draft.pdf (accessed 1 October 2020).

\section{Part 1: EMSSA emergency point-of- care ultrasound training guidelines Introduction}

Training is often cited as the biggest potential barrier to the successful implementation of widespread accurate emergency pointof-care ultrasound (ePoCUS) use. ${ }^{[1,2]}$ To train and maintain the skills required by ePoCUS users, a significant commitment needs to be made toward the provision of high-quality courses countrywide, along with readily accessible supervised practical exposure. Furthermore, a robust competency and credentialing system with continuous governance and quality control is vital. ${ }^{[3,4]}$

\section{Principles of training}

EMSSA has followed the same basic training phases as highlighted by the International Federation for Emergency Medicine (IFEM). ${ }^{[4]}$ The typical training process in developing a new skill follows four phases (Fig. 1):

1. An initial induction phase. This phase is in the form of an introductory course, which consists of a theoretical component followed by hands-on practical exposure, i.e. the development of both cognitive and psychomotor skills required to successfully perform emergency point-of-care ultrasound (ePoCUS). The theoretical component will be completed online and the 'flipped classroom' approach will be used (Table 1).

2. A development-of-skills phase. This is when the individual gains experience in the clinical and practical use of ePoCUS through ongoing use on patients in daily clinical practice. This should ideally be done under appropriate supervision to ensure the user learns and is remediated appropriately (Table 2). EMSSA supports the role of self-directed adult learning in this phase of skills development. In the past, it had been assumed that the completion of a specified number of ultrasound scans would result in competence in that specific ePoCUS application. Various programmes, ${ }^{[3,5]}$ including EMSSA's previous guidelines, ${ }^{[6]}$ required a minimum number of scans in a particular application before it is possible for a candidate to proceed to a formal credentialing assessment. Although these requirements might aid in producing competence, their use as a single criterion for triggering an assessment of competence is limited and fails to take into account the principle of individualised learning and variable learning curves. ${ }^{[7]}$ Therefore, EMSSA recommends a certain number of scans be completed for each application but will not enforce it nor require a formal logbook submission prior to attempting the credentialing examination. Some candidates will become competent with a lower number of scans, while others might require more practice to develop competency (Table 3).

3. Proof-of-competency phase. Competence for each ePoCUS application will be assessed based on three main areas of proficiency: knowing when to use a specific ePoCUS application; successful ability to acquire and optimise ultrasound images; and correct interpretation of the ePoCUS exam findings along with the successful integration of these into individual patient care plans.
This will be assessed at the credentialing exam as detailed in Part 2 of this policy statement.

4. Ongoing training. It is vital that all ePoCUS users appreciate the importance of continued and regular utilisation of each ePoCUS application to avoid losing the skills gained. This is especially applicable to those who have already been successfully credentialed. EMSSA recommends keeping an up-to-date logbook of all ePoCUS examinations performed, even after the credentialing process. EMSSA will also run a minimum of one ePoCUS-based continuing medical education (CME) activity per year to ensure ePoCUS providers are being kept up to date and continuing their learning. This will take the form of participation in EMSSA roadshows, symposia or dedicated ultrasound simulation sessions and webinars or refresher courses.

\section{Training faculty}

The executive committee of the EMSSA PoCUS SIG, according to its constitution, consists of a chair, a secretary and regional representatives. Ideally, all nine provinces in the country should have regional representation. The current working situation is that the main provincial representatives are supporting the non-represented regions of the country, namely:

- Northern Cape is represented by the Western Cape faculty.

- Free State is represented by the Eastern Cape faculty.

- Mpumalanga is represented by the KwaZulu-Natal faculty.

- North West is represented by the Gauteng (Pretoria) faculty.

- Limpopo is represented by the Gauteng (Johannesburg) faculty.

EMSSA recommends that there should be a clinical lead for each application in both core and advanced curricula. The clinical lead will be a member of the EMSSA ePoCUS SIG and will be responsible for all matters regarding the particular application as well as the regular updating of the guidelines and curriculum as new evidence emerges.

\section{Training guidelines for core curriculum \\ Course structure}

The newly revised core curriculum, which consists of six key modules, is described in more detail in Part 3 of the policy. In order to continually improve medical education and ensure that it is relevant and up to date, EMSSA has revised the existing 2009 ePoCUS curriculum. This is to achieve a more competence-based and individualised learning structure while still achieving standardised outcomes as set out by international ePoCUS training societies.

One way in which EMSSA has approached this is by splitting the course into theoretical and practical components. The existing didactic lecture series used in the 1-day core course has been converted to a flipped classroom style of teaching (Table 1). This consists of a series of online video presentations covering each of the core modules. Each video presentation will include sections on:

- How to perform the core ePoCUS application including image acquisition and optimisation.

- The application's limitations and common pitfalls.

- The correct use of the application in a clinical context.

This will allow individuals to progress through the theoretical content of each module at their own pace, ensuring maximal uptake of vital information. Following each module, the content will be further reinforced by a set of MCQs and VAQs, which must be passed before the learner will be allowed to move on to the next module. This further ensures that the module content has been fully understood by the learner before they can progress. Completion of all online 


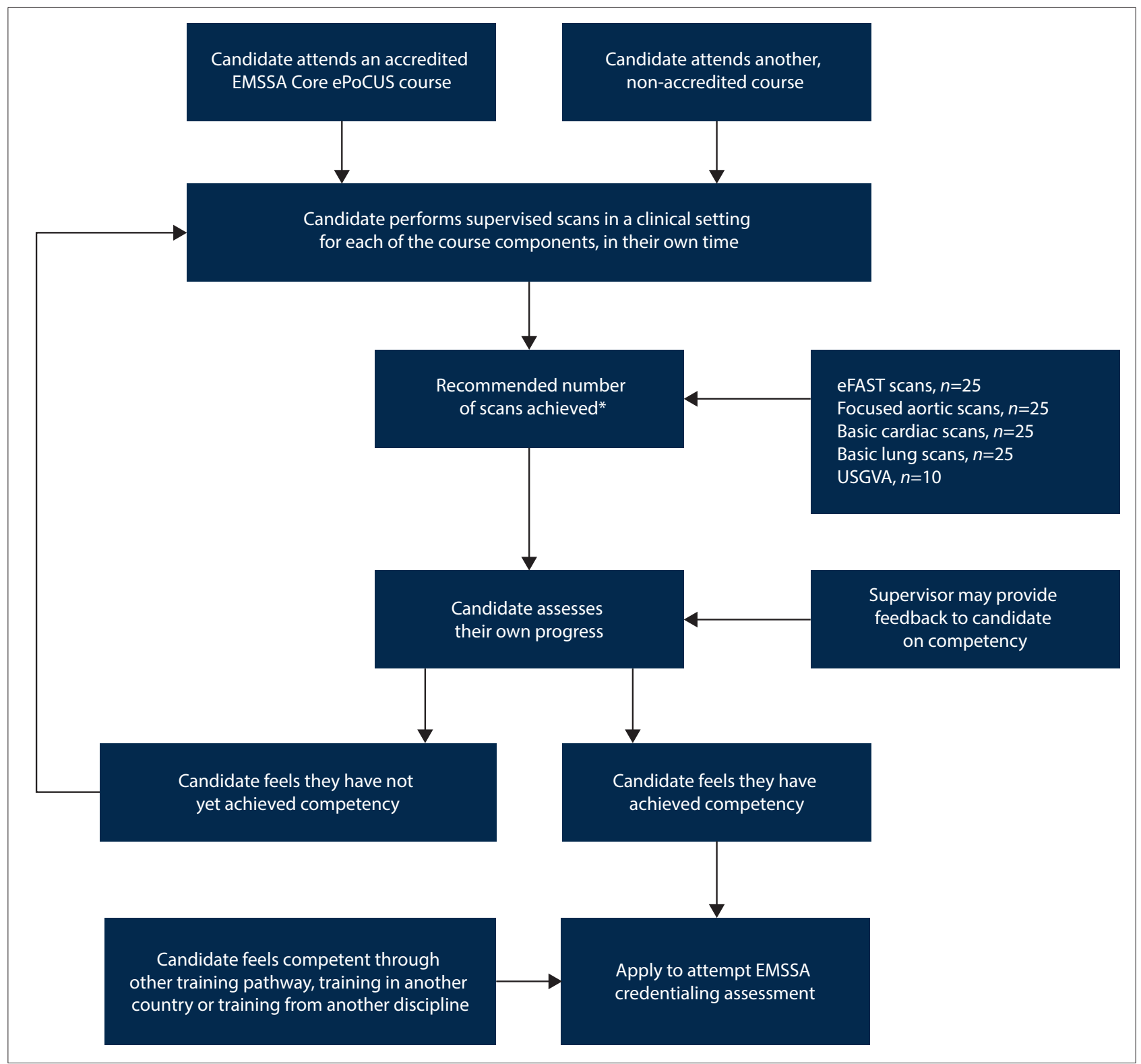

Fig. 1. Pathway for training and credentialing in emergency point-of-care ultrasound. (EMSSA = Emergency Medicine Society of South Africa; ePoCUS = emergency point-of-care ultrasound; eFAST = extended focused assessment with sonography in trauma; USGVA = ultrasound-guided vascular access.) ( ${ }^{*}$ This number does not necessarily have to be achieved to trigger a competency assessment.)

modules is compulsory before proceeding to the hands-on practical training component.

The practical course will be run over a 1-day period and real-time scanning of live simulated patients will be used for teaching and practice. There will be interactive, question-based reviews of the theory needed for each module during the hands-on practical. This is to ensure that maximal time is dedicated to teaching and learning of the crucial psychomotor skills required for ePoCUS. This training will be facilitated by experienced ePoCUS instructors, who have gone through specific ePoCUS instructor training (see below). A ratio of no more than five learners to one instructor will be used to ensure personalised learning for each candidate. Ensuring good instructor to learner ratios will ensure more focused learning groups and improved skill uptake. ${ }^{[8]}$

For the training on ultrasound-guided vascular access skills, EMSSA recommends the use of vascular phantoms, which mimic real-life sono-anatomy and provide similar tactile feedback to the real-life experience. This will provide the participants with the opportunity for multiple attempts and allow them to master the skill.

\section{Course criteria}

The following criteria must be adhered to for each course:

- A large venue to accommodate all learners and instructors comfortably as well as to provide space for scanning stations with a stretcher or examination bed at each station.

- The venue should have appropriate audio-visual set up to allow for visual presentations and real-time master demonstrations.

- Each ultrasound scanning station should consist of an examination bed or stretcher, an appropriate ultrasound machine with all required transducers, ultrasound gel and paper towels.

- A maximum ratio of five learners to one instructor must be maintained. 


\section{Table 1. The flipped classroom concept}

The flipped classroom is an educational strategy, based on blended learning, in which the focus of information transmission is outside of the classroom and in-classroom time is spent on exploring topics in greater depth and ensuring that learning has occurred. The focus becomes the learner rather than the teacher. This system allows for better management of candidates with mixed levels of knowledge, experience and different learning preferences. It also allows for a greater time to be spent on learning practical skills rather than information.

In the setting of EMSSA ultrasound courses, the learner will study and assimilate the necessary curriculum information from online material prior to the course. The course itself will then focus on formative assessments of knowledge and facilitation of understanding. The course will also, and most importantly, provide instruction on the practical aspects of ultrasound usage - the training of hand-probe-eye coordination. A substantial amount of time is provided for the learner to practise the skills themselves while receiving direct and immediate feedback.

\section{Table 2. Supervision of clinical scanning}

Appropriate supervision is important at all levels of learning ePoCUS. This supervision is important for two main reasons: firstly, to assist and train in the acquisition and optimisation of appropriate images, video clips or measurements in real patients under a variety of clinical circumstances; and secondly, to ensure that the candidate can interpret and apply the findings correctly in the clinical context in which the scan is performed.

For this reason, the ideal supervisor would be a senior emergency medicine doctor who is an EMSSA-credentialed instructor in the skill being supervised. Unfortunately, it will not always be possible for this to happen. Therefore, other practitioners who are proficient in the skill being learnt may supervise scans. Supervision of the technical component of the scanning may be done by any experienced person (including sonographers) as long as they are familiar with the exact EMSSA requirements for each type of scan. However, instruction relating to the interpretation of the scans and incorporating these findings into the clinical decision matrix needs to be provided by an appropriate clinician experienced in ePoCUS.

\section{Table 3. Logbooks}

It is essential for practitioners of ePoCUS at all levels to keep a logbook of the scans that they have performed or supervised. Although the details of a candidate's scanning history will not determine their eligibility to attend initial credentialing assessment, the logbook is important for several reasons:

1. The contents of the logbook can be used as part of formative assessments during the training process.

2. The logbook can be used to justify application for and entry to advanced ePoCUS course components.

3. The logbook is most important for re-credentialing. A scanning history that is consistent with regular clinical use of ePoCUS will allow a candidate to re-credential with an abbreviated assessment rather than a full credentialing assessment.

4. A documented scanning history is evidence of experience in the skills documented, which has both positive academic and medico-legal significance.

The logbook should not include patient names or identifying information. Each entry should contain the patient's gender, age, brief information on the indication for scan, scan performed and findings. It should also include whether the scan was performed or supervised.

- Learners should rotate to a different scanning station for each module, ensuring exposure to a variety of ultrasound machines, instructors and simulated patients.

- Simulated patients with normal anatomy should be used at each scanning station.

- Informed consent must be obtained from all patient models. A waiver of liability related to unexpected underlying pathology should also be signed. If any undiagnosed pathology is noted in any of the simulated patients, it is the responsibility of the course coordinator to inform them and assist with appropriate medical referral, if necessary.

- A course feedback form needs to be completed and returned by all course attendees on the day of the course.

\section{Trainee instructor selection criteria}

In order to ensure the provision of high-quality ePoCUS training across SA, a dedicated teaching faculty is essential. Training of faculty as new instructors requires good governance to firstly ensure that the correct content is being taught in a standardised and evidence-based manner, and secondly to ensure that the trainee instructor is adhering to good teaching principles. A candidate will need to be nominated by two certified ePoCUS instructors to become an EMSSA-accredited course trainee instructor. The motivation for the nomination can include, but is not limited to, their performance at the credentialing exam, an aptitude for ePoCUS in daily clinical practice and/or an eagerness to teach ePoCUS. They will also require current core EMSSA credentialing certification within the last 4 years or evidence of regular scanning (an updated logbook with at least 100 clinically indicated scans per year). Each nomination will need to be approved by the EMSSA PoCUS SIG faculty.

\section{Instructor criteria and maintenance of instructorship}

To become a core EMSSA course instructor, a nominee must instruct and teach on a minimum of two courses as a candidate instructor. During each of these courses, the candidate instructor will be assessed on their ability to perform interactive questionbased teaching in addition to practical bedside teaching for each of the course modules. A standardised assessment form will be used for this purpose and will be completed by the course coordinator (Appendix 1; http://samj.org.za/public/sup/15346.pdf). Feedback 
should be provided to the candidate instructor by the course coordinator, highlighting areas where they excelled as well as areas that require improvement or remediation. The completed assessment forms need to be submitted timeously to the EMSSA PoCUS SIG secretary and chair. Only once the two sets of assessment forms have been reviewed and approved by the PoCUS SIG can a candidate instructor be certified as a full EMSSA course instructor. A certificate will be issued to each new instructor, valid for a 2-year period. A biennial renewal of instructorship will be required.

To ensure eligibility for renewal and maintenance of instructor status, the following conditions must be met:

- Instructors must maintain their credentials as ePoCUS providers.

- Instructors must instruct on a minimum of two EMSSA core ultrasound courses (or equivalent) or examine on a minimum of two credentialing examinations per year, or a combination thereof.

- Instructors must keep a current logbook reflecting a minimum of 100 clinically indicated ePoCUS examinations per year.

- Instructors will be formally assessed by a course coordinator on the quality of their instruction during one of the courses on which they are teaching.

- The logbook and formal assessment will need to be sent to the EMSSA PoCUS SIG secretary for approval by the SIG faculty on a biennial basis.

If a previous instructor has not instructed in the last 2 years and/or has failed to provide a satisfactory logbook demonstrating regular clinical use of ePoCUS, they will need to re-credential as per the normal instructor credentialing pathway.

\section{Core course coordinator criteria}

For an EMSSA core course instructor to become a course coordinator they will need:

- To be a member of the EMSSA PoCUS SIG management committee.

- To have instructed on a minimum of four core ultrasound courses as a full instructor, one of which they coordinated under supervision of a credentialed course coordinator.

- All new course coordinators require approval by the PoCUS SIG.

\section{Core curriculum candidate examiner for credentialing}

Candidate examiners will be nominated and approved by the EMSSA SIG faculty. Candidates must be course instructors or coordinators, with at least 2 years' experience. In order for a candidate examiner to become an approved examiner, they will have to observe and assist on two credentialing examinations and be endorsed by the chief examiners from those courses.

\section{Training guidelines for advanced curriculum \\ Course structure}

Please refer to Part 4 of the policy statement for a more detailed explanation of the advanced course curriculum. Training in advanced ultrasound is contingent upon completing core ultrasound education, training and credentialing as per the core ePoCUS guidelines.

The principles of training and learning for each module of the advanced curriculum are the same as discussed in the core curriculum training process. The initial induction phase will share the same online format as the core course. The three compulsory and two optional modules, chosen by the learner, will be made available online. These can be completed in the candidate's own time. This will then be followed by a hands-on practical course in which the learner will be given the opportunity to perform scanning on live models or phantoms under the supervision of experienced advanced ePoCUS instructors. The course would be customised to the specific modules chosen by the candidates. This phase of training will be similar to that of the core curriculum training, but in the advanced curriculum, the responsibility of gaining experience in each module will be driven largely by the learner. Support and guidance during this period can be sought from the various EMSSA clinical leads assigned to each advanced ePoCUS application.

Competency in advanced ePoCUS will be assessed according to the same principles as the core course. However, each module in the advanced curriculum will be credentialed and examined separately.

\section{Candidate instructor criteria for the advanced course}

Criteria to become an advanced ePoCUS candidate instructor include:

- Nomination in writing to the EMSSA SIG chair by two ePoCUS instructors from different regions.

- Approval by PoCUS SIG faculty.

- The nominee must be a current EMSSA core ultrasound course instructor (meeting the criteria of an up-to-date instructor as highlighted above).

- The nominee must be a current EMSSA core course credentialing examiner (within the last 2 years).

- The nominee must provide evidence of regular scanning in the required advanced applications (current logbook with at least 100 clinically indicated scans per year).

- Alternatively, the nominee should have an equivalent diploma or fellowship in ePoCUS approved by the SIG faculty.

\section{Advanced instructor criteria and maintenance of instructorship}

The candidate instructor will have to attend a minimum of two advanced curriculum courses at which an assessment of their bedside teaching skills and overall knowledge on the advanced applications will be assessed using a standardised assessment form (Appendix 1). In order to maintain instructor status, the instructor will have to teach or examine a minimum of one EMSSA advanced ultrasound course or credentialing examination per year.

\section{Advanced credentialing examiner}

All EMSSA PoCUS SIG faculty approved advanced course instructors are encouraged to become examiners. The requirement to become registered as an advanced credentialing examiner will be to observe and assist on one advanced credentialing course exam.

\section{Advanced course coordinator criteria}

The process to coordinate an advanced ultrasound course requires significant ePoCUS experience. The criteria required to become an advanced course coordinator are:

- To be a member of the EMSSA SIG faculty.

- To be a current EMSSA core course coordinator.

- To have instructed on a minimum of four advanced ultrasound courses, one of which was coordinated under supervision.

- All new course coordinators require prior approval by the EMSSA PoCUS SIG.

\section{Non-EMSSA PoCUS courses}

As the value of and need for ePoCUS skills continue to be recognised in all disciplines of medicine, the demand for training will increase. ${ }^{[9]}$ As a result, there will be an increase in the number of courses being run by other organisations, societies and private for-profit companies. 
EMSSA supports and encourages the principle of advancing the widespread teaching and training of ePoCUS, provided the quality and content of this training is appropriate.

EMSSA does not endorse any particular course other than its own. All content from the online course component and the practical day remains the property of EMSSA. Accreditation and licensing of nonEMSSA ePoCUS courses is a significant task and EMSSA does not have the capacity to be the accreditation body for all ePoCUS courses in the country.

However, EMSSA will remain the sole organisation for credentialing ePoCUS in SA in order to maintain standards of proficiency and standardisation of evaluation of candidates. An individual who has attended a non-EMSSA ePoCUS course will be permitted to attempt the EMSSA ePoCUS credentialing examination (see Part 2 for further details). However, given the nature of this examination, we would recommend that candidates have:

- attended an appropriate training course

- performed appropriate scans for each course

- made use of ePoCUS in the clinical setting under the direction of an appropriate supervisor.

\section{Training pathways}

\section{Emergency medicine registrar training}

Since the last EMSSA policy statement in 2009, it has been a mandatory requirement for all emergency medicine registrars to be credentialed in core (formerly level 1) ePoCUS before being allowed to attempt the final FCEM (SA) part II examinations. This still remains a requirement by the College of Emergency Medicine and a recommendation by EMSSA. Furthermore, registrars may be expected to demonstrate their knowledge and skill in ePoCUS in both the written and practical components of the emergency medicine specialty examinations.

Following registrar attendance at a core ePoCUS course, it is vital that the emergency medicine training programme assists with continued training and education on ePoCUS. EMSSA recommends appointing a dedicated member of university academic staff, with experience and interest in PoCUS and education to oversee matters related to the use of ePoCUS in the emergency medicine department. This person would be responsible for coordinating, planning and conducting all PoCUS teaching essential to ensure successful training of all emergency medicine registrars in the programme. Various educational ePoCUS teaching activities that are recommended could include:

- Dedicated bedside teaching utilising ePoCUS in the various EDs. This would require the identification of PoCUS trainers/instructors in each of the training units to ensure that this is achieved.

- Specific departmental academic days dedicated to ePoCUS teaching. This could be in the form of lectures, presentations and hands-on practical experience utilising patient models and/or phantoms.

- Mock examination and simulation cases should be run regularly to assess an individual's competence in the various core ePoCUS applications.

- Regular journal clubs to review and appraise the latest ePoCUS literature.

\section{Training for other doctors}

Not all potential users of ePoCUS will be registrars nor will they be working within academic departments. Nonetheless, the skills required will be the same to pass the credentialing assessment. EMSSA will facilitate training for these candidates by running training courses in addition to formal courses. However, each department in which candidates are preparing for credentialing should run a training programme similar to that described for academic units.

\section{Alternative training and credentialing for allied disciplines}

With the increasing uptake of ePoCUS across all fields of clinical medicine, there is a corresponding increased and diverse need for ePoCUS training in other disciplines allied to emergency medicine. ${ }^{[10-12]}$

Emergency medical services providers, nurses and clinical associates

The utility of ePoCUS is not only limited to doctors but can be valuable to other non-doctor healthcare workers as well. There is evolving literature on the beneficial role that ePoCUS plays in both the prehospital and nursing environments. ${ }^{[13-15]}$ The basic principles of the EMSSA guidelines should be adhered to by the respective ultrasound faculties wishing to set up an ePoCUS training programme, if they wish to make use of the EMSSA credentialing process. The basic training process should include a similar structure to the doctors' pathway, i.e. an initial induction phase introducing the core concepts of ePoCUS and the applications with practical experience, followed by a training phase, and finally, overall competence assessment in the form of a credentialing exam.

Specific discussions and decisions will be needed regarding the specific learning and environmental needs as well as what ultrasound applications will be necessary and beneficial to the specific nondoctor groups. Each discipline should develop their own curriculum based on their needs. This will be supported by EMSSA.

Clinical associates are mid-level healthcare providers who primarily work under the supervision of a qualified medical practitioner and have been doing so in SA since 2011. ${ }^{[16]}$ Equivalent to physician assistants in the USA, they possess the skills necessary for the prevention, diagnosis and management of common medical conditions. ${ }^{[17]}$ Therefore, ePoCUS is seen as a useful and necessary skill in their training and EMSSA recommends the development of a training programme specific to the needs of their curriculum.

\section{Other specialties}

While there are similarities in the needs for PoCUS among various other medical specialties and emergency medicine, there are inherent differences in the pathology, practice and training. Therefore, while a generalised PoCUS training for all specialties would add benefit, EMSSA believes there are specialty-specific applications required for each discipline. Each specialty should be responsible for developing their own specific guidelines, utilising the principles set out in this policy statement. Collaboration between all specialties in the training of PoCUS is crucial to ensure high-quality bedside scanning and improved patient safety.

Notwithstanding the independence of each speciality, EMSSA has the strong opinion that there should be a PoCUS credentialing process in place for each of these specialties without exception. The failure of other specialties to have established these processes is not in keeping with good academic principles, practice and patient safety.

\section{Medical interns}

Due to the lack of any formal PoCUS exposure at an undergraduate level or during internship, the majority of newly qualified doctors in SA start their medical careers without knowing how to perform and 
utilise PoCUS in their daily practice. ${ }^{[18]}$ The overall benefit PoCUS can provide to the training of junior doctors with regards to their clinical skills, diagnostic and procedural capabilities, and overall patient management cannot be underestimated. ${ }^{[19]}$ EMSSA believes that a lack of any formal training in PoCUS during internship would be seriously detrimental to junior doctors' development into competent independent healthcare providers. Furthermore, dedicated PoCUS training objectives should be set out in the internship training guidelines. The most appropriate allocation would be in their family medicine block, where they have dedicated emergency medicine time. EMSSA does not limit access to its courses or credentialing process based on years of experience and encourages all doctors to learn the skills of PoCUS as early on in their careers as possible.

\section{Future considerations}

\section{Medical student training}

In several medical schools in the United States and Canada, emergency medicine has been at the forefront in improving undergraduate medical education through the early integration of PoCUS in clinical training. ${ }^{[20]}$ The early integration of PoCUS at an undergraduate level has been shown to improve medical students' understanding of anatomy and pathology, and their physical examination and clinical skills. ${ }^{[19,20]}$ EMSSA strongly recommends the development of a PoCUS training programme at an undergraduate level. The key would be to identify a qualified individual who will be the champion of undergraduate PoCUS training.

It is clear that medical students and interns training would require a fundamental change in the Health Professions Council of South Africa (HPCSA) guidelines. Training in these skills will become increasingly important; however, these changes will need to be motivated for by universities and other interested organisations.

\section{Fellowship training}

The American College of Emergency Physicians (ACEP), in a policy statement in 2016, stated that 'fellowships provide the advanced training needed to create future leaders in an evolving area of medicine., ${ }^{[3]}$ ePoCUS certainly is a rapidly evolving area of clinical medicine and the development of a local fellowship training programme will only serve to enhance and promote this. Wellestablished ePoCUS fellowships in emergency medicine exist in Canada and the United States, but no established programmes exist on the African continent. ${ }^{[21,22]}$ EMSSA believes that establishing a fellowship programme in SA will not only have a positive impact on the overall ePoCUS usage, but will serve to strengthen existing ePoCUS training programmes. It will also speed up the development of new programmes within emergency medicine and other specialties, both locally and further afield. Furthermore, it will act as a catalyst to promote and drive research in the field of ePoCUS, with the overall goal of improved patient care through its utilisation.

\section{Conclusion}

EMSSA is committed to the provision of high-quality and up to date ePoCUS training. We will continue to increase our training capacity and broaden our training exposure geographically and clinically while at the same time continuing to strengthen our networks both locally and internationally. This will, we hope, ensure the widespread uptake and use of ePoCUS across SA with the resultant improvement in overall patient care.
1. Micks T, Sue K, Rogers P. Barriers to point-of-care ultrasound use in rural emergency departments. CJEM 2016;18(6):475-479. https://doi.org/10.1017/cem.2016.337

2. Shah S, Bellows BA, Adedipe AA, Totten JE, Backlund BH, Sajed D. Perceived barriers in the use of ultrasound in developing countries. Crit Ultrasound J 2015;7(1):28. https://doi.org/10.1186/s13089ultrasound in

3. American College of Emergency Physicians (ACEP). Emergency ultrasound guidelines. Irving: ACEP, 2016. https://www.acep.org/patient-care/policy-statements/ultrasound-guidelines-emergency-point2016. https://www.acep.org/patient-care/policy-statements/ultrasound-guidelines-emergency-pont-
of-care-and-clinical-ultrasound-guidelines-in-medicine/ (accessed 1 October 2020). . Atkinson P, Bowra J, Lambert M, Lamprecht H, Noble V, Jarman B. International Federation for Emergency Medicine point-of-care ultrasound curriculum. CJEM 2015;17(2):161-170. https://doi. org/10.1017/cem.2015.8

5. Australasian College for Emergency Medicine (ACEM). Policy on the use of bedside ultrasound by emergency physicians. Melbourne: ACEM, 2006. https://acem.org.au/getmedia/0702004f-c6694646-b5fc-4fa577117ba3/COR742_v1_ED_Ultrasound_Training_Governance_Guideline (accessed 1 October 2020).

6. Wells M, Bruijns S. College of Emergency Medicine of South Africa policy document. Emergency ultrasound in South Africa. Johannesburg: EMSSA, 2009. https://emssa.org.za/documents/ CEM(SA)-Emergency-Ultrasound-POLICY.pdf (accessed 1 October 2020).

Blehar DJ, Barton B, Gaspari RJ. Learning curves in emergency ultrasound education. Acad Emerg Med 2015;22(5):574-582. https://doi.org/10.1111/acem.12653

8. Safavi AH, Shi Q, Ding M, et al. Structured, small-group hands-on teaching sessions improve pre-clerk knowledge and confidence in point-of-care ultrasound use and interpretation. Cureus 10(10):e3484. https://doi.org/10.7759/cureus.3484

9. Moore CL, Copel JA. Point-of-care ultrasonography. N Engl J Med 2011;364(8):749-757. https://doi. org/10.1056/NEJMra0909487

10. LoPresti CM, Schnobrich DJ, Dversdal RK, Schembri F. A road map for point-of-care ultrasound training in internal medicine residency. Ultrasound J 2019;11(1):10. https://doi.org/10.1186/ s13089-019-0124-9

11. McCormick TJ, Miller EC, Chen R, Naik VN. Acquiring and maintaining point-of-care ultrasound (PoCUS) competence for anaesthesiologists. Lacquisition et le maintien des compétences en (PoCUS) competence for anaesthesiologists. Lacquisition et le maintien des compétences en
échographie au chevet (PoCUS) pour les anesthésiologistes. Can J Anaesth 2018;65(4):427-436.
https://doi org/10.1007/s12630-018-1049-7

12. Meadley B, Olaussen A, Delorenzo A, et al. Educational standards for training paramedics in Meadley B, Olaussen A, Delorenzo A, et al. Educational standards for training paramedics in
ultrasound: A scoping review. BMC Emerg Med 2017;17(1):18. https://doi.org/10.1186/s12873-017-

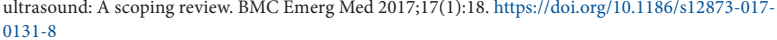
$0131-8$

Bøtker MT, Jacobsen L, Rudolph SS, Knudsen L. The role of point-of-care ultrasound in prehospital critical care: A systematic review. Scand J Trauma Resusc Emerg Med 2018;26(1):51. https://doi. org/10.1186/s13049-018-0518-x

14. Bahl A, Pandurangadu AV, Tucker J, Bagan M. A randomised controlled trial assessing the use of ultrasound for nurse performed IV placement in difficult access ED patients. Am J Emerg Med 2016;34(10):1950-1954. https://doi.org/10.1016/j.ajem.2016.06.098

15. Hutchings S, Bisset L, Cantillon L, et al. Nurse-delivered focused echocardiography to determine intravascular volume status in a deployed maritime critical care unit. J R Nav Med Serv 2015;101(2):124-128. https://doi.org/10.1186/2197-425x-3-s1-a919

16. Doherty J, Couper I, Fonn S. Will clinical associates be effective for South Africa? S Afr Med J 2012;102(11 Pt 1):833-835. https://doi.org/10.7196/samj.5960

17. Mittman DE, Cawley JF, Fenn WH. Physician assistants in the United States. BMJ 2002;325(7362):485487. https://doi.org/10.1136/bmj.325.7362.485

18. Health Professions Council of South Africa (HPCSA). Handbook on internship training: Guidelines for interns, accredited facilities and health authorities. Pretoria: HPCSA, 2017. https://www. hpcsa-blogs.co.za/wp-content/uploads/2017/04/2017-IN-Handbook-Part-I-and-II.pdf (accessed 1 October 2020).

19. Solomon SD, Saldana F. Point-of-care ultrasound in medical education - stop, listening and look. N Engl J Med 2014;370(12):1083-1085. https://doi.org/10.1056/NEJMp1311944

20. Cook T, Hunt P, Hoppman R. Emergency medicine leads the way for training medical students in clinician-based ultrasound: A radical paradigm shift in patient imaging. Acad Emerg Med 2007;14(6):558-561. https://doi.org/10.1197/j.aem.2007.04.003

21. Canadian Society of Point-of-Care Emergency Medicine Fellowships. POCUS, 2014. http://pocus. Canallow Society of Point-of-Care Emergen
calloccessed 1 October 2020).

2. Society of Clinical Ultrasound Fellowships. SCUF, 2020. https://eusfellowships.com/program-listnew/ (accessed 1 October 2020)

\section{Part 2: Credentialing in emergency point-of-care ultrasound Background}

There is near-universal agreement that clinicians who wish to use PoCUS should undergo some form of credentialing process before they are permitted to do so independently, as a matter of patient safety. ${ }^{[1]}$ There is also consensus that the endpoint of the credentialing process should be to establish that the user is competent in the theoretical and practical application of the elements of PoCUS assessed. ${ }^{[2]}$

While there are limited data on the best method of training ePoCUS, it is clear that different routes to competency are possible and the best routes may differ between individuals. How training and learning occurs is therefore less important than the achievement of competency. ${ }^{[3]}$ EMSSA still recommends a certain method for training based on currently available evidence. However, for the purposes of credentialing, no evidence of the training process will need to be provided. There will be no specified course and no stipulated minimum number of scans to qualify for credentialing. The credentialing process will be robust enough to ensure that competency in ePoCUS has been achieved by those who are successful at the credentialing examination. 
There are significant benefits to having a national credentialing system. ${ }^{[4]}$ This system allows for uniformity of standards across the country as well as across differing healthcare facilities. It also allows and requires healthcare providers in different disciplines to achieve the same level of competence in ePoCUS skills. ${ }^{[5]}$

There is still a need for hospital-based recognition of privileges. This is particularly important to allow different specialties to recognise which of their providers are credentialed to perform PoCUS. It is also important that different disciplines are able to recognise the scopes of practices and limitations of how PoCUS is used outside of their own discipline. In addition, EMSSA believes that it is essential for other disciplines including cardiology, obstetrics and gynaecology and trauma surgery to adopt a formal credentialing system to ensure quality and safety in the use of PoCUS within their own fields.

The details of the processes are described below in the following sections:

- Who is eligible to attempt the credentialing process?

- What is the process to register for credentialing?

- What is the process for credentialing?

- What if a candidate is not successful in credentialing?

- What needs to be done to maintain credentialing?

- What differs between credentialing for core ePoCUS and advanced ePoCUS?

\section{Who is eligible to attempt the credentialing process?}

Any independent practitioner may attempt the credentialing examination. There are no specific training or experiential requirements, and experience through self-directed learning is appropriate. However, given the nature of the examination, we would recommend that candidates have:

- Attended an appropriate training course.

- Performed at a minimum the recommended number and type of scans for each course.

- Made use of PoCUS in the clinical setting under appropriate supervision.

\section{What is the process to register for credentialing?}

Bookings for the credentialing examination may be made through the EMSSA website or by contacting the EMSSA secretary directly. Once the fee for credentialing has been received, the candidate will then be offered a place on the next available credentialing session.

\section{What is the process for credentialing?} Philosophy of the examination

The primary objective of the credentialing examination is to ensure that candidates have all the skills required to use ePoCUS safely and effectively. This would include:

- The necessary knowledge.

- Competent psychomotor skills (image generation and hand-probeeye coordination for procedural guidance).

- The ability to interpret images appropriately.

- The ability to incorporate the PoCUS findings correctly into the clinical decision-making matrix.

The credentialing process should ideally be separated from the training process. Whenever possible, the persons responsible for training the candidates should not be involved in their examination to avoid conflicts of interest and to ensure an impartial assessment by disinterested and neutral examiners.

Finally, the credentialing process should be transparent, with the process and broad content of the exams known to the candidates. This not only allows for a fair examination process but, since candidates are likely to focus on material which will be examined, will improve the level of skills developed by candidates. In essence, the candidates should know what is expected from them to be able to show competency and to ensure that they are competent in performing these skills.

\section{Format and components of the examination}

There will be two components to the credentialing process: an MCQ/ VAQ examination and an examination with simulated patients. Both these examinations will be conducted on the same day. The details of each component of the competency assessment are shown in Table 4.

The MCQ/VAQ examination will cover the entire scope of the ePoCUS components including the ability to identify both normal and abnormal findings. The simulation examination will similarly cover all the components including image generation and procedural skills. These simulations will extend beyond simply demonstrating the ability to acquire images but will evaluate the candidate's ability to select the correct scans to perform, to correctly identify the findings and how these will impact on further management.

\section{Assessment of the outcome of the examination}

The organisation of the examination and the assessment of the outcome are shown in Fig. 2. The following criteria will be used to assess the outcome of the written and practical examinations:

Table 4. The format of the credentialing examination

Examination component

Skills assessed

Theoretical knowledge

Image interpretation

Identification of normal and abnormal findings

Cases with simulated patients

(3 simulation stations, 20 min per station)
Incorporation of ultrasound into the clinical decision matrix

Theoretical knowledge

Image generation and optimisation

Image interpretation

Identification of normal and abnormal findings

Incorporation of ultrasound into the clinical scenario and the clinical

decision matrix

Procedural skills 


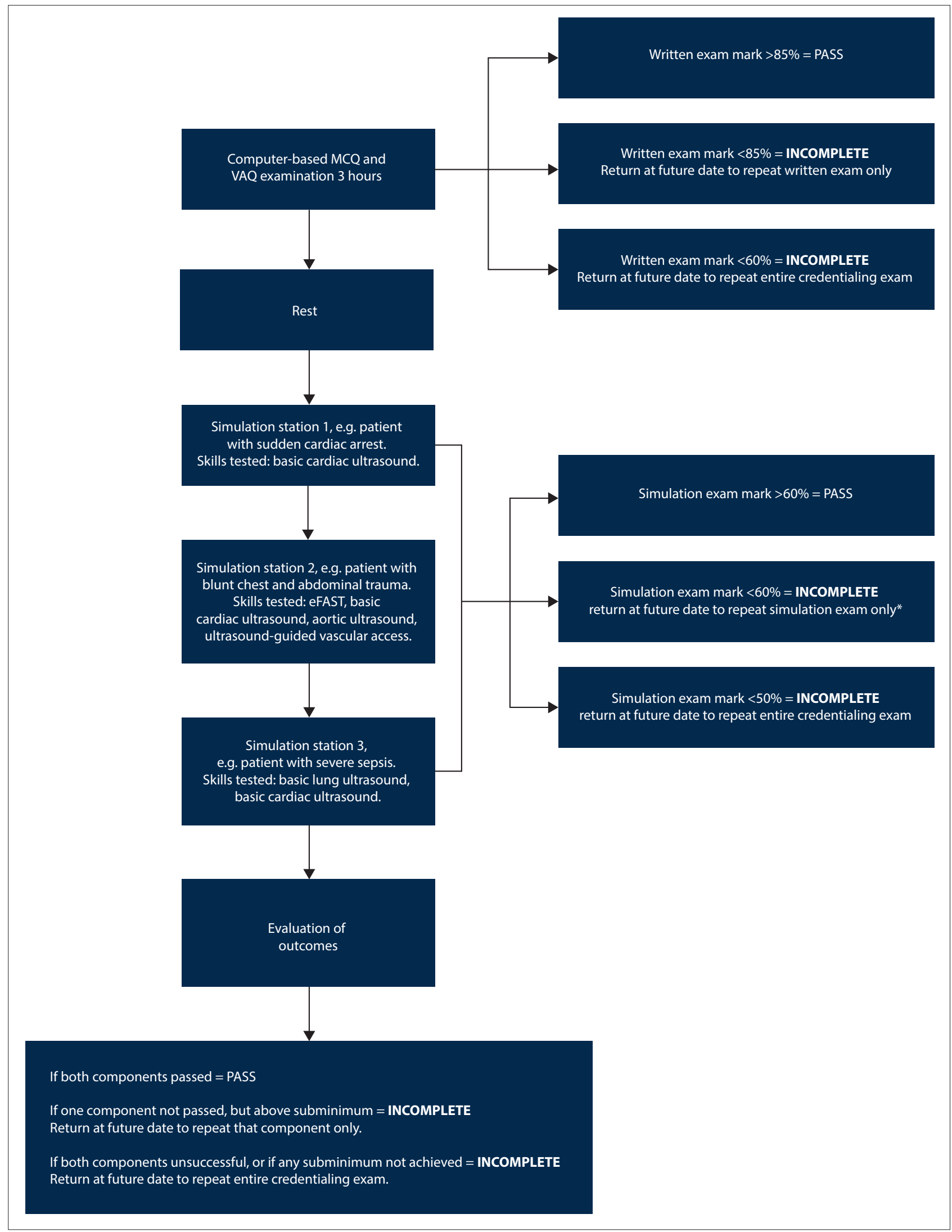

Fig. 2. Flow diagram illustrating the examination process and assessment of outcomes. (MCQ = multiple-choice questions; VAQ = visual-aid questions; eFAST = extended focused assessment with sonography in trauma.)

$\left({ }^{*}\right.$ A candidate may be offered an opportunity to perform an additional simulation station if they are unsuccessful with one of the simulated patients. The simulated scenario will be different, but the skills tested will be similar.) 
- The pass mark for the MCQ/VAQ examination will be $85 \%$.

- The pass mark for each simulation examination will be $60 \%$.

- The candidate will need to pass both the MCQ/VAQ examination and each simulation examination to be successful at the credentialing.

- If the candidate passes one component but not the other, the outcome will be regarded as an 'incomplete - repeat component' and they may return at a subsequent credentialing examination to repeat that component.

- If a candidate is unsuccessful in a single simulation station, but achieved a mark of more than $50 \%$, they may be invited to attempt a supplementary simulation station at the same credentialing session.

- If a candidate failed the MCQ/VAQ examination and one or more of the simulation stations, the outcome shall be regarded as 'incomplete - repeat credentialing examination' and the candidate will need to reattempt the entire examination.

- In addition, certain additional subminimum rules apply:

- A candidate receiving a mark of less than $60 \%$ in the MCQ/VAQ examination will need to return to repeat the entire examination. A candidate receiving a mark of less than $40 \%$ in any one of the simulation cases will need to return to repeat the entire examination.

\section{Location and frequency of examinations}

Examinations will be run at central locations (presently Johannesburg, Cape Town, Durban, Pretoria and Gqeberha) across SA at regular intervals, with each region hosting at least one credentialing session per year.

\section{Examiner panel}

Examiners, who are required to be experienced sonologists and EMSSA instructors, will be nominated from each of the regions and approved by the executive committee of the PoCUS SIG of EMSSA. Examiners will be appointed for each of the credentialing examination sessions at the beginning of each year. Regional credentialing examinations should have at least one external examiner from a different geographical region to ensure the consistency and standard of the examination process and conduct.

\section{Cost of the examination}

There is a significant cost associated with conducting credentialing examinations. The prices will be available on the EMSSA website or can be obtained from the secretary of EMSSA. Candidates who need to repeat the entire credentialing examination will need to register and pay for the repeat assessment; candidates who may need to repeat a component of the credentialing will need to register and pay a reduced fee.

\section{What if a candidate is not successful in credentialing?}

If a candidate received an 'incomplete - repeat component' assessment, they will need to return at a future credentialing session to reattempt the component of the examination at which they were not successful. If a candidate received an 'incomplete - repeat credentialing examination' assessment they will need to return to reattempt the entire credentialing examination.

\section{What needs to be done to maintain credentialing?}

All clinicians using ePoCUS need to undergo a credentialing assessment every 4 years. If an already credentialed provider is able to produce a logbook with evidence of having performed at least 100 clinically indicated scans per year for each of the previous 4 years, then they may register for an abbreviated credentialing assessment. All other candidates must reattempt the full credentialing examination. Users who are credentialed as advanced ePoCUS providers will only need to recredential as advanced providers and not core providers as well. The abbreviated recredentialing examination will consist of three simulated patient stations. During the 15-minute simulation, both theoretical knowledge and practical skills will be assessed, which will cover the full spectrum of the appropriate curriculum. These abbreviated recredentialing examinations will be charged at a reduced rate and may also be booked on the EMSSA website.

\section{What differs between credentialing for core ePoCUS and advanced ePoCUS?}

The credentialing process will be similar for the core ePoCUS module and the advanced ePoCUS modules. During the examination for core ePoCUS, all components of the curriculum will be examined using multiple examination techniques (eFAST, basic lung ultrasound, basic cardiac ultrasound, vascular access and basic aortic scanning). Multiple components will be examined during each simulation station. During the examination for advanced ePoCUS, each module will be examined separately, but also using multiple examination techniques.

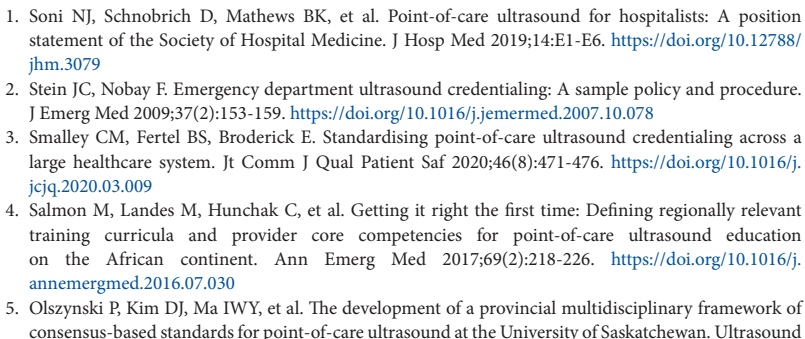
large healthcare system. Jt Comm J Qual Patient Saf 2020;46(8):471-476. https://doi.org/10.1016/j. jcjq.2020.03.009

4. Salmon M, Landes M, Hunchak C, et al. Getting it right the first time: Defining regionally relevan training curricula and provider core competencies for point-of-care ultrasound education on the African continent. Ann Emerg Med 2017;69(2):218-226. https://doi.org/10.1016/j. annemergmed.2016.07.030

5. Olszynski P, Kim DI, Ma IWY, et al. The development of a provincial multidisciplinary framework of consensus-based standards for point-of-care ultrasound at the University of Saskatchewan. Ultrasound J 2019;11(1):28. https://doi.org/10.1186/s13089-019-0142-7

\section{Part 3: Curriculum for core emergency point-of-care ultrasound training Introduction}

Emergency point-of-care ultrasound (ePoCUS) can be defined as ultrasound performed at the bedside by the treating clinician to provide diagnostic and procedural guidance in the overall management of a patient in the emergency setting. ${ }^{[1]}$ Several terms have been used to describe this form of bedside ultrasound, which is distinct from the formal, consultative ultrasound performed by sonographers and radiologists. These include terms such as bedside, emergency, focused, clinical, and physician-performed ultrasound; however, the term that is all encompassing, most widely accepted and adopted is that of PoCUS. ${ }^{[2]}$

Several studies have shown that the utilisation of ePoCUS has led to an improvement in the quality of emergency care provided by clinicians with regards to procedural safety and diagnostic accuracy, reducing length of stay in hospital and overall costs. ${ }^{[3-6]}$ With its well-established role in emergency care, being proficient in ePoCUS is essential for all clinicians dealing with critically ill patients. It is recommended by the majority of emergency medicine societies across the world. ${ }^{[1,7-10]}$ The inclusion of ultrasound training in general emergency medicine curricula and specialty examinations is widely encouraged. South Africa's College of Emergency Medicine requires competency in core ePoCUS before emergency medicine registrars 
are permitted to attempt their final exams. ${ }^{[8]}$ Emergency medicine remains the only discipline in SA to require an approved training process and formalised credentialing in PoCUS.

The specialty of emergency medicine has taken a leadership role in the establishment and training of ePoCUS use by clinicians across the globe, with SA being no exception. ${ }^{[1,11]}$ EMSSA and its PoCUS SIG recognises the significant role played by ePoCUS in the overall diagnosis and management of patients presenting to $\mathrm{ED}$ across southern Africa. EMSSA has been responsible for ePoCUS training, credentialing and its clinical governance in SA for the past 12 years and has certified core ePoCUS proficiency in well over 100 healthcare providers to date.

It is important to continue this process of training in ePoCUS and the credentialing of its users, but it is also important to remain clinically up-to-date and evidence-based. The last EMSSA policy document released on ePoCUS in SA was in 2009. ${ }^{[8]}$ This revised and updated EMSSA position statement provides modernised guidance on the use of ePoCUS, with recommendations on curricula, scope of practice, training and credentialing, and record-keeping and good clinical governance.

There are several position statements and established ePoCUS curricula available from countries that have established emergency medicine training programmes. EMSSA recognises the unique circumstances in which we practise emergency medicine in SA and has therefore tailored its training curricula and credentialing mechanisms to reflect this.

EMSSA has previously divided its ultrasound training programme into level 1 and level 2 PoCUS curricula. This nomenclature is nondescriptive and not in line with other international curricula. EMSSA has therefore changed the nomenclature to core and advanced ePoCUS curricula.

In general, the criteria used for the inclusion of applications into the core ePoCUS curriculum were: $:^{9,11]}$

- The technique must be easy to learn, perform and interpret.

- It must have widespread utility.

- There must be a significant evidence base to support its use.

- It must be important in emergency diagnosis or management.

- It should have a significant impact on burden of disease, resources, morbidity and mortality profiles in SA.

However, advanced ePoCUS curriculum applications are generally: ${ }^{[9]}$

- More difficult to learn, perform and/or interpret.

- Have a more limited utility in terms of the number of patients benefitting from the scans.

- Are usually more time consuming than core applications.

- Have less of an impact in a SA context.

\section{Purpose and scope of practice}

This document is a policy statement of the EMSSA and relates to the use of core and advanced ultrasound at the bedside in the EDs both in the public and private healthcare sectors. Other specialties such as critical care or anaesthesia may use this document as reference, provided they are performing the ePoCUS exam in the manner described.

\section{Ultrasound applications}

The following ePoCUS applications have been recommended by EMSSA as essential to the practice of emergency medicine in SA. Many other major international emergency medicine societies echo the prioritisation of these applications as core curriculum components. It is therefore recommended that all healthcare providers working in an emergency setting or dealing with emergencies on a regular basis, attain or actively strive towards attaining these core ePoCUS skills:
- Technical skills such as image acquisition and optimisation.

- Extended focused assessment with sonography in trauma (eFAST).

- Focused abdominal aorta ultrasound.

- Basic cardiac ultrasound.

- Basic lung ultrasound.

- Ultrasound-guided vascular access.

EMSSA recognises that those working in emergency settings may use several other ultrasound applications. Their non-inclusion as basic applications does not lessen their importance nor imply that treating clinicians are unable to use them in daily practice.

Notably, the assessment for features or presence of DVTs using limited compression ultrasound has been removed as a core application. This is in keeping with most major international curricula, as well as being in line with the criteria defining a core PoCUS application. EMSSA recognises the value of being able to perform this procedure and has included it in the advanced curriculum.

\section{Image acquisition and optimisation}

A critical component of mastering PoCUS is ensuring the best possible image quality is achieved at all times. The only way to accomplish this is to have a good basic understanding of the physics of ultrasound and how an ultrasound machine works. Furthermore, recognising and understanding how various ultrasound artefacts are generated is vital. This will ensure accurate interpretation of the images generated and in so doing, assist in the best bedside diagnosis possible.

\section{Required competencies}

- Basic knowledge of ultrasound physics.

- Basic knowledge of how ultrasound images are generated.

- An understanding of the interaction of ultrasound with tissues.

- The ability to recognise and explain common artefacts.

- An understanding of the different ultrasound transducers and indications for use.

- An understanding of the key components of an ultrasound machine's controls ('knobology') including overall gain, time gain compensation, depth and focus point.

- The ability to optimise image generation.

- An understanding of the different ultrasound modes: B-mode, M-mode and Doppler ultrasound (pulse wave and colour wave).

\section{Extended focused assessment with sonography in trauma (eFAST)}

The eFAST exam is performed mainly, but not exclusively, in the trauma setting to detect haemoperitoneum, haemopericardium, haemothorax and pneumothorax. It can be further extended to the haemodynamically unstable patient to rapidly rule out acute intraabdominal blood loss as a cause for the shocked state, most notably in patients with a ruptured abdominal aortic aneurysm or ruptured ectopic pregnancy. It is an ePoCUS application that is used on a daily basis in the SA setting, with a significant impact on patient management decisions, particularly in resource-limited environments.

\section{Required competencies}

- An understanding of the principles and the indications of the eFAST exam.

- An understanding of the concept and the limitations of a 'rule-in' philosophy.

- The ability to recognise pitfalls in eFAST imaging (e.g. artefacts and free fluid mimics). 
- The ability to incorporate ultrasound findings with the rest of the clinical findings.

- The ability to image the six key areas entirely:

- Right upper quadrant view: all four areas in this view need to be visualised, i.e. above the right hemidiaphragm, between the right hemidiaphragm and the liver, between the liver and the right kidney (Morison's pouch) and the inferior pole of the right kidney/caudal tip of the liver.

- Left upper quadrant view: all four areas in this view need to be visualised, i.e. above the left hemidiaphragm, between the left hemidiaphragm and the spleen, between the spleen and the left kidney and the inferior pole of the left kidney.

- Subcostal/subxiphoid view: the entire pericardium is visualised. Use of the parasternal long axis view of the heart as an alternative to visualise the heart and its pericardium.

- Pelvic views: imaging in both transverse and longitudinal planes to assess for the presence of free fluid in rectovesical pouch (males) or recto-uterine pouch (females).

Bilateral pleural views: to assess for the presence or absence of lung sliding and to be able to identify the 'lung-point sign' to diagnose the presence of a pneumothorax.

\section{Recommended number of scans}

- A recommended minimum of 25 accurate and clinically indicated eFAST exams.

- At least $50 \%$ of the scans should demonstrate pathology.

- All scans should ideally be supervised or reviewed by an experienced and trained ePoCUS provider.

\section{Focused abdominal aorta ultrasound assessment}

A focused abdominal aorta ultrasound involves scanning of the aorta in both longitudinal and transverse planes. Scanning should be done from the epigastrium down to the aortic bifurcation to visualise both common iliac arteries, assessing for the presence of an aneurysm or a dissection. Imaging the abdominal aorta is seen as a core application based on its utility in an emergency setting with the potential to have a significant impact on diagnosis and management of patients presenting with undifferentiated shock. It is rapid to perform and answers a simple binary question.

\section{Required competencies}

- The ability to assess the abdominal aorta in its entirety in both transverse and longitudinal views.

- The ability to recognise pitfalls in scanning the abdominal aorta (bowel gas, adipose tissue) and develop skills to overcome these limitations.

- The ability to accurately measure the abdominal aorta in at least three areas in longitudinal plane (proximal, mid and distal) and at least one in the transverse plane.

- The ability to recognise the different forms and sites of aneurysms and the importance therefore of imaging in two planes.

- The ability to identify aneurysmal dimensions $>5 \mathrm{~cm}$ (those with a high risk of rupture), as well as features of aortic dissection.

- The ability to identify key landmark vessels originating from the abdominal aorta (coeliac trunk, superior mesenteric artery and common iliac arteries).

- The ability to identify the inferior vena cava and recognise key features differentiating it from the abdominal aorta.

- The ability to interpret the images in the correct clinical context and extend ePoCUS applications where relevant, i.e. performance of an eFAST exam to assess for haemoperitoneum if concerned for a ruptured or leaking abdominal aortic aneurysm.

\section{Recommended number of scans}

- A recommended minimum of 25 accurate and focused abdominal aorta scans.

- At least $50 \%$ of the scans should be clinically indicated.

- All scans should ideally be supervised or reviewed by an experienced and trained ePoCUS provider.

\section{Basic cardiac ultrasound}

The ability to image the heart using transthoracic echocardiography provides a wealth of information in the diagnosis and management of the haemodynamically unstable, peri-arrest or cardiac arrest patient. ${ }^{[12]}$ In the haemodynamically unstable, peri-arrest patient, focused cardiac ultrasound can assist with differentiating the cause of the shock, and guiding the necessary interventions and treatment required. ${ }^{[12]}$ Several recent expert consensus statements on basic cardiac ultrasound highlight the important role it plays in rapidly and accurately assisting with the identification of the pathophysiology of shock and directing appropriate treatment. ${ }^{[12-14]}$ Focused echocardiographic evaluation in resuscitation (FEER) is one of the terms used to describe the use of basic cardiac ultrasound primarily in a cardiac arrest situation. ${ }^{[15]}$ Due to the importance of continuous high-quality cardiopulmonary resuscitation (CPR) in cardiac arrest, it is vital that the timing of a FEER examination does not interfere with CPR, and should either occur during the initial patient assessment or after five cycles of high-quality CPR. Cardiac ultrasound in this scenario is used to rapidly identify reversible causes for the cardiac arrest, guide the quality of chest compressions and accurately assess for the presence or absence of cardiac activity ${ }^{[16]}$ It is important to note that basic cardiac ultrasound is not a comprehensive echocardiography exam and cannot replace this.

\section{Required competencies}

- The ability to use basic cardiac ultrasound appropriately and to integrate findings in the clinical context.

- An understanding of and the ability to perform a FEER protocol appropriately.

- The ability to image the heart in its standard echocardiographic planes:

- Parasternal long-axis view

- Parasternal short-axis view

Apical four-chamber view

Subxiphoid view

It is recognised that not all the views will be necessary or sometimes be achievable for all patients. It is, however, always prudent to image the heart in at least two planes before drawing conclusions regarding pathology.

- An understanding of probe selection, probe orientation and patient positioning to generate the best cardiac images.

- The ability to identify major cardiac pathology:

- The presence or absence of cardiac activity.

The presence of pericardial fluid.

- Gross chamber size abnormalities.

- Basic gestalt assessment of left ventricular systolic function.

- The ability to estimate intravascular fluid status (cardiac views, lung and inferior vena cava imaging).

\section{Recommended number of scans}

- A recommended minimum of 25 accurate and clinically indicated basic cardiac ultrasound exams.

- At least $50 \%$ of the scans should show pathology.

- All scans should ideally be supervised or reviewed by an experienced and trained ePoCUS provider. 


\section{Basic lung ultrasound}

One of the most valuable ePoCUS skills all doctors treating medical emergencies should acquire, is the ability to perform lung ultrasound. Its ease of use, rapidity and reliability along with absence of radiation make it a far better imaging modality than standard chest X-rays for the diagnosis and management of chest pathology in emergencies. This includes the identification of a pneumothorax, haemothorax, pulmonary oedema, pleural effusions and pneumonias. ${ }^{[1718]}$ Lung ultrasound is a surface imaging technique used to image the pleura and the artefacts generated from this layer, to give an indication of what is occurring within the thoracic cavity. Lung ultrasound is widely viewed as one of the easiest-to-learn PoCUS applications as it is not technically demanding, answers simple binary questions and does not require specific, challenging acoustic windows. ${ }^{[17,19]}$

\section{Required competencies}

- The ability to integrate lung ultrasound findings with clinical context and other ePoCUS modalities (focused cardiac ultrasound and inferior vena cava findings).

- An understanding and recognition of the limitations and pitfalls of lung ultrasound.

- The ability to utilise a standardised approach to imaging the lungs:

- Correct probe orientation and selection for the type of lung pathology being investigated (linear probe for pleural pathology, curvilinear for B-lines).

- Standard view to include two rib shadows with hyperechoic pleural line located below the ribs.

- Imaging in a systematic fashion, dividing the thorax into specific zones.

- The ability to recognise the most important artefacts arising from the pleural line and understand their meaning/significance:

- A-lines: horizontal hyperechoic reverberation artefacts.

B-lines: vertical hyperechoic lines.

- The ability to recognise and understand the importance of lungsliding as well as the sonographic features and meaning of a lungpulse sign and a lung-point sign.

- The ability to utilise M-mode to assess and document the presence/ absence of lung sliding.

- The ability to recognise and diagnose common basic lung pathology:

- Pneumothorax: presence of A-lines, absence of lung sliding potentially with a lung-point sign.

- Pulmonary oedema: presence of multiple B-lines in multiple lung zones bilaterally

- Pleural effusion: anechoic fluid located in dependant areas of the thorax.

- Pneumonia: recognise pleural irregularities, and variations of lung consolidation including the 'shred' sign.

\section{Recommended number of scans}

- A recommended minimum of 25 correctly performed and clinically indicated lung ultrasound exams.

- At least $50 \%$ of the scans should demonstrate pathology including a range of conditions, e.g. pneumothorax, pleural effusion, pulmonary oedema and pneumonia.

- All scans should ideally be supervised or reviewed by an experienced and trained ePoCUS provider.

\section{Ultrasound-guided vascular access}

Procedural ultrasound is the use of an ultrasound device to guide and enhance the success of a medical intervention. ${ }^{[1]}$ Its utility is far reaching, from guiding peripheral vein cannulation to thoracocentesis, pericardiocentesis, lumbar punctures and nerve blocks. By identifying relevant anatomy and pathology before proceeding with an invasive procedure, the accuracy and complication rates are improved. ${ }^{[20]}$ It is important to note that procedural ultrasound is a vital adjunct to emergency care.

EMSSA has included ultrasound-guided vascular access as a core application due to its extensive benefit in daily emergency medicine practice. Other forms of procedural ultrasound are included in the advanced curriculum.

Difficult peripheral vascular access and the insertion of central venous catheters are common procedures in the ED. The ultrasound examinations for these procedures are performed at the bedside to identify vascular anatomy and guide direct visualisation and cannulation of vessels. This has been shown to improve patient safety, decrease the number of complications, increase first-time success rates, and decrease the number of attempts and time to perform the cannulation. ${ }^{[21-24]}$ Many of the major international emergency medicine organisations have emphasised ultrasoundguided central venous catheter insertion as a key intervention that should be implemented immediately into the care of all patients. ${ }^{[25-27]}$ The British National Institute for Health and Care Excellence (NICE) guideline on central venous catheter placement recommends the use of ultrasound guidance in most clinical circumstances where central venous catheter insertion is necessary either electively or in an emergency situation. In addition, they recommend that all those involved in placing central venous catheters should undertake appropriate training to achieve competence. ${ }^{[25]}$

\section{Required competencies}

- An understanding of the limitations and contraindications to performing ultrasound-guided vascular access.

- A sound understanding of the relevant vascular sono-anatomy:

- The ability to differentiate an artery from a vein.

- The ability to identify relevant large central veins (internal jugular, subclavian, femoral).

- The ability to identify relevant large peripheral veins (basilic and cephalic).

- An understanding of the ability to utilise the correct machine settings, probe, probe orientation and patient positioning to ensure the best possible imaging.

- The ability to optimise the machine and sonographer positioning to ensure ideal ergonomics and ease of procedure.

- An understanding of when to use the two possible imaging techniques used in ultrasound-guided vascular access and their advantages and limitations:

- Static: anatomic structures and an insertion position are identified with ultrasound. The procedure then proceeds as it would without ultrasound and is not performed with the transducer imaging the patient through key components of the procedure.

- Real-time: the key components of the procedure are performed with simultaneous ultrasound visualisation during the procedure (e.g. using ultrasound to visualise a needle entering a vessel).

- An understanding of when to use the two possible imaging planes used in ultrasound-guided vascular access and their advantages and limitations:

- Out-of-plane: a short-axis, transverse approach images the vessel and needle in cross section and therefore only a hyperechoic dot is represented by the needle on the ultrasound screen.

- In-plane: a longitudinal, long-axis approach allows the entire length and angle of the vessel and needle to be visualised. 
- An understanding of and ability to apply the principles of sterility with regards to central venous catheter insertion.

- An ability to cannulate both peripheral and central vessels using both in-plane and out-of-plane orientations.

\section{Recommended number of scans}

- A recommended minimum of 10 successful and clinically indicated ultrasound-guided central venous catheter insertions.

- A recommended minimum of 10 successful and clinically indicated ultrasound-guided peripheral vein cannulations.

- All scans should ideally be supervised or reviewed by an experienced and trained ePoCUS provider.

\section{Training and credentialing}

Please refer to parts 1 and 2 of the guidelines.

\section{Conclusion}

The newly updated and revised core ePoCUS curriculum serves to provide an evidence-based approach to providing and maintaining a standard of care in PoCUS in emergency medicine in the SA context.

Contributions of the authors. All authors contributed equally to this work.

1. American College of Emergency Physicians (ACEP). Emergency ultrasound guidelines. Irving: ACEP, 2016. https://www.acep.org/patient-care/policy-statements/ultrasound-guidelines-emergency-point2016. https://www.acep.org/patient-care/policy-statements/ultrasound-guidelines-e
of-care-and-clinical-ultrasound-guidelines-in-medicine/ (accessed 1 October 2020).

2. Alonso JV, Turpie J, Farhad I, Ruffino G. Protocols for point-of-care-ultrasound (PoCUS) in a patient with sepsis: An algorithmic approach. Bull Emerg Trauma 2019;7(1):67-71. https://doi. org/10.29252/beat-0701010

Shokoohi H, Boniface KS, Pourmand A, et al. Bedside ultrasound reduces diagnostic uncertainty and guides resuscitation in patients with undifferentiated hypotension. Crit Care Med 2015;43(12):25622569. https://doi.org/10.1097/CCM.0000000000001285

4. Durston W, Carl ML, Guerra W. Patient satisfaction and diagnostic accuracy with ultrasound by emergency physicians. Am J Emerg Med 1999;17(7):642-646. https://doi.org/10.1016/s07356757(99)90150-x

5. Lindelius A, Törngren S, Nilsson L, Pettersson H, Adami J. Randomised clinical trial of bedside ultrasound among patients with abdominal pain in the emergency department: Impact on patient ultrasound among patients with abdominal pain in the emergency department: Impact on patient
satisfaction and health care consumption. Scand J Trauma Resusc Emerg Med 2009;17:60. https:// satisfaction and health care cons
doi.org/10.1186/1757-7241-17-60

6. Smallwood N, Dachsel M. Point-of-care ultrasound (PoCUS): Unnecessary gadgetry or evidencebased medicine? Clin Med 2018;18(3):219-224. https://doi.org/10.7861/clinmedicine.18-3-219
bat

based medicine? Clin Med 2018;18(3):219-224. https://doi.org/10.7861/clinmedicine.18-3-219
. Australasian College for Emergency Medicine (ACEM). Policy on the use of bedside ultrasound by emergency physicians. Melbourne: ACEM, 2006. https://acem.org.au/getmedia/0702004f-c6694646-b5fc-4fa577117ba3/COR742_v1_ED_Ultrasound_Training_Governance_Guideline (accessed 1 October 2020)

8. Wells M, Bruijns S. College of Emergency Medicine of South Africa policy document. Emergency Ultrasound in South Africa. Johannesburg: EMSSA, 2009. https://emssa.org.za/documents CEM(SA)-Emergency-Ultrasound-POLICY.pdf (accessed 1 October 2020).

9. Atkinson P, Bowra J, Lambert M, Lamprecht H, Noble V, Jarman B. International Federation for Emergency Medicine point-of-care ultrasound curriculum. CJEM 2015;17(2):161-170. https:// doi:10.1017/cem.2015.8

10. Canadian Association of Emergency Physicians (CAEP). Position statement: Recommendations for the use of point-of-care ultrasound (PoCUS) by emergency physicians in Canada. Ottawa: CAEP, 2019. http://caep.ca/wp-content/uploads/2019/08/CAEP-PoCUS-Position-Statement-2019-Full2019. https://caep.ca/wp-conten
text.pdf (accessed 25 April 2020).

11. Van Hoving DJ, Lamprecht $H$. Toward an appropriate point-of-care ultrasound curriculum: A reflection of the clinical practice in South Africa. J Med Ultrasound 2016;24:18-24. https://doi. org/10.1016/j.jmu. 2015.11 .00

12. Via G, Hussain A, Wells M, et al. International evidence-based recommendations for focused cardiac ultrasound. J Am Soc Echocardiogr 2014;27(7):683.e1-683.e33. https://doi.org/10.1016/j. echo.2014.05.001

13. Labovitz AJ, Noble VE, Bierig M, et al. Focused cardiac ultrasound in the emergent setting: A consensus statement of the American Society of Echocardiography and American College of Emergency Physicians. J Am Soc Echocardiogr 2010;23(12):1225-1230. https://doi.org/10.1016/j. echo.2010.10.005

14. Vincent JL, Rhodes A, Perel A, et al. Clinical review: Update on hemodynamic monitoring consensus of 16. Crit Care 2011;15(4):229. https://doi.org/10.1186/cc10291

15. Breitkreutz R, Walcher F, Seeger FH. Focused echocardiographic evaluation in resuscitation Breitkreutz R, Walcher F, Seeger FH. Focused echocardiographic evaluation in resuscitation
management: Concept of an advanced life support-conformed algorithm. Crit Care Med 2007;35(5 Suppl):S150-S161. https://doi.org/10.1097/01.CCM.0000260626.23848.FC

16. Gardner KF, Clattenburg EJ, Wroe P, Singh A, Mantuani D, Nagdev A. The cardiac arrest sonographic assessment (CASA) exam - a standardised approach to the use of ultrasound in PEA. Am J Emerg Med 2018;36(4):729-731. https://doi.org/10.1016/j.ajem.2017.08.052

17. Mayo PH, Copetti R, Feller-Kopman D, et al. Thoracic ultrasonography: A narrative review. Intensive Care Med 2019;45(9):1200-1211. https://doi.org/10.1007/s00134-019-05725-8

18. Lichtenstein DA, Mezière GA. Relevance of lung ultrasound in the diagnosis of acute respiratory failure: The BLUE protocol. Chest 2013;144(2):721 https://doi.org/10.1378/chest.07-2800

19. Strnad M, Zadel S, Klemenc-Ketis Z, Prosen G. Identification of lung sliding: A basic ultrasound technique with a steep learning curve. Signa Vitae 2013;8(1):31-35. https://doi.org/10.22514/ SV81.052013.5
20. Peabody CR, Mandavia D. Deep needle procedures: Improving safety with ultrasound visualisation. J Patient Saf 2017:13(2):103-108. https://doi.org/10.1097/PTS.0000000000000110

1. Randolph AG, Cook DJ, Gonzales CA, Pribble CG. Ultrasound guidance for placement of central venous catheters: A meta-analysis of the literature. Crit Care Med 1996;24(12):2053-2058. https://doi. org/10.1097/00003246-199612000-00020

22. Hind D, Calvert N, McWilliams R, et al. Ultrasonic locating devices for central venous cannulation: . Hind D, Calvert N, McWilliams R, et al. Ultrasonic locating devices for central ve
Meta-analysis. BMJ 2003;327(7411):361. https://doi.org/10.1136/bmj.327.7411.361

23. Egan G, Healy D, O’Neill H, et al. Ultrasound guidance for difficult peripheral venous access: Systematic review and meta-analysis. Emerg Med J 2013;30(7):521-526. https://doi.org/10.1136 emermed-2012-20165

24. Stolz LA, Stolz U, Howe C, Farrell IJ, Adhikari S. Ultrasound-guided peripheral venous access: A metaanalysis and systematic review. J Vasc Access 2015;16(4):321-326. https://doi.org/10.5301/jva.5000346

25. National Institute for Health and Care Excellence (NICE). Guidance on the use of ultrasound locating devices for placing central venous catheters. London: NICE, 2002. https://www.nice.org.uk/guidance/ ta49/resources/guidance-on-the-use-of-ultrasound-locating-devices-for-placing-central-venouscatheters-pdf-2294585518021 (accessed 1 October 2020).

26. American Society of Anaesthesiologists Task Force on Central Venous Access, Rupp SM, Apfelbaum JL, et al. Practice guidelines for central venous access: A report by the American Society of Anaesthesiologists task force on central venous access. Anaesthesiology 2012;116(3):539-573. https:// Anaesthesiologists task force on central ve
doi.org/10.1097/ALN.0b013e31823c9569

27. Troianos CA, Hartman GS, Glas KE, et al. Guidelines for performing ultrasound guided vascular cannulation: Recommendations of the American Society of Echocardiography and the Society of Cardiovascular Anesthesiologists. J Am Soc Echocardiogr 2011;24(12):1291-1318. https://doi. org/10.1016/j.echo.2011.09.021

\section{Part 4: Curriculum for advanced emergency point- of-care ultrasound training Introduction}

In SA, the first formalised use of PoCUS was described in 2008 in an article published in the $S A M J$ by members representing all the then divisions of emergency medicine countrywide. ${ }^{[1]}$ This was followed by the release of a policy document by the College of Emergency Medicine of South Africa on ePoCUS in SA. ${ }^{[2]}$ These publications introduced the curriculum for basic training in ePoCUS. Although EMSSA has provided advanced emergency ePoCUS training in SA for the last decade, no recognised or standardised curriculum has been promulgated. This is partly because no such curriculum exists in other parts of the world and partly because the skills to train and credential advanced techniques have not been available. This practice guideline is the first to detail the curriculum and requirements for advanced ePoCUS practice in SA.

The advanced curriculum will expand on the core ePoCUS skills. In keeping with international guidelines, those areas of ePoCUS which are beyond the core level will be expanded upon in this document with regards to guidance on the knowledge, training and competencies expected. ${ }^{[3-7]}$ Training in advanced ultrasound is contingent upon core ultrasound education, training and credentialing having been completed as per the core ePoCUS guidelines (see parts 2 and 3 ).

The intention of the advanced ePoCUS credentialing is that a doctor who has achieved this qualification should be considered an advanced ultrasound practitioner, i.e. one who can self-learn and selfteach, and would not need further credentialing in new or additional individual PoCUS applications or skills, with an exception of specific components such as advanced echocardiography.

\section{Purpose and scope of practice}

This document is a policy of the EMSSA and relates to the use of advanced ePoCUS in the (ED). The policy is applicable to both public and private hospital ED throughout SA. It is recommended that other disciplines who make use of ePoCUS should adhere to these policies or those produced by their own speciality. Guidelines that apply to ultrasound examinations or procedures performed by specialists in other departments, or in a different setting, may not be applicable to emergency physicians. ${ }^{[4]}$

The following modules have been included in the advanced ePoCUS curriculum:

- Focused cardiac ultrasound and haemodynamic assessment.

- DVT extended compression ultrasound. 
- Advanced thoracic (lung) and airway ultrasound.

- Regional anaesthesia and nerve blocks.

- Hepatobiliary and genito-urinary tract PoCUS.

- Gastrointestinal tract PoCUS.

- Focused obstetric and gynaecological PoCUS

- Transcranial Doppler and ocular ultrasound.

This list reflects the current practice of advanced ePoCUS. It will be reviewed periodically as further areas of practice become recognised as part of the scope of PoCUS.

\section{Applications/modules}

In order to obtain the advanced ePoCUS credentialing, proficiency needs to be demonstrated in a minimum of five modules. Three of the modules will be compulsory:

- Focused cardiac ultrasound and haemodynamic assessment.

- DVT extended compression ultrasound.

- Advanced thoracic (lung) and airway ultrasound.

The remaining two choices of advanced modules can be made from the following options:

- Regional anaesthesia and nerve blocks.

- Hepatobiliary and genito-urinary tract PoCUS.

- Gastrointestinal tract PoCUS.

- Focused obstetric and gynaecological PoCUS

- Transcranial Doppler and ocular ultrasound.

\section{Focused cardiac ultrasound and haemodynamic assessment}

Focused cardiac ultrasound or focused echocardiography and the ability to be able to evaluate the haemodynamic status of a patient are crucial skills for doctors working in the ED. It is more advanced than basic cardiac ultrasound but still is not a comprehensive echocardiographic examination. These skills can be utilised in but are not limited to the following patient presentations: ${ }^{[3,8]}$

- The dyspnoeic patient in whom an isolated cardiac or respiratory case is not clinically obvious or to evaluate the acutely dyspnoeic patient presenting with pulmonary oedema.

- The patient with undifferentiated shock.

- The peri-arrest patient or the patient in cardiac arrest.

- Suspected massive pulmonary embolism.

- The evaluation of patients with chest pain.

- The evaluation of patients with abnormal cardiovascular findings on clinical examination, abnormal radiological findings or abnormal electrocardiogram findings.

\section{Required competencies}

- To be able to describe the indications and limitations of focused cardiac ultrasound.

- To demonstrate a knowledge of normal cardiac ultrasound anatomy.

- To be competent in the acquisition of standard echocardiographic views: parasternal long- and short-axis views; apical four- and fivechamber views; subcostal view including the inferior vena cava; and suprasternal view.

- To be able to recognise common important cardiac pathologies:

- Pericardial effusion (including tamponade) and the appearance of pleural effusions on echocardiography.

- Reduced ejection fraction (systolic function).

- Cardiac chamber dilation including the different types of cardiomyopathy.

- Gross wall motion abnormalities.
- Recognition of the features of massive pulmonary embolism including evaluation of pulmonary pressures.

- Gross valve abnormalities (regurgitation, stenosis and features of infective endocarditis).

- To be able to assess gross left ventricular systolic function using visual estimation and E-point septal separation.

- To be able to assess gross right ventricular systolic function using visual estimation, tricuspid annular plane systolic excursion (TAPSE) and tissue Doppler imaging.

- To be able to assess left ventricular diastolic function.

- To be able to evaluate stroke volume using left ventricular outflow tract velocity-time integral (LVOT VTI) as well as aortic flow respiratory variability.

- To be able to use M-mode, colour flow Doppler, tissue Doppler imaging (TDI) and spectral Doppler modalities such as pulse wave Doppler and continuous wave Doppler.

- To be able to evaluate for thoracic aortic dilation and dissection using standard transthoracic views.

\section{Recommended number of scans}

- At least 1 year of experience at core level with a total of no less than 250 logged ePoCUS scans (of any type).

- A recommended minimum of 50 correctly performed, clinically indicated focused cardiac ultrasound exams.

- At least $50 \%$ of the scans should demonstrate pathology (including a range of conditions).

- All scans should ideally be supervised or reviewed by an experienced and trained ePoCUS provider.

\section{DVT extended compression ultrasound}

PoCUS can be used to evaluate patients who are suspected of having a lower-limb DVT. The principal component of the evaluation is the bilateral compressibility of the proximal deep veins extending all the way from the common femoral vein, through the popliteal vein to the confluence of the calf veins. ${ }^{[3]}$ This differs from limited compression ultrasound (where two or three short segments are examined), in that the entire length of the femoral vein is visualised and tested for compressibility.

Assessment for DVT should include:

- Application of the appropriate risk-stratification pathway, e.g. Wells DVT score.

- Awareness of the ultrasonographic appearance of the following: - Acute DVT.

- Superficial thrombophlebitis.

- Lymph nodes.

- Duplicate veins.

- Arterial aneurysms and calcified arteries.

- Popliteal cysts.

- Awareness of the limitations of compressibility PoCUS with regards to:

- Patient factors (e.g. morbid obesity, grossly oedematous limbs).

- Proximal vein evaluation in high-risk patients (inferior vena cava assessment).

Specific competencies for DVT evaluation include:

- To be able to describe the indications and limitations of PoCUS for the detection of DVT.

- To be able to identify the common femoral vein at the level of the inguinal ligament and follow the vein distally to the confluence of the superficial femoral vein and the deep femoral vein.

- To be able to assess the lumen of the vein with respect to size, intraluminal echogenicity and compressibility. 
- To be able to identify and follow the superficial femoral vein distal to the inguinal ligament.

- To be able to assess the lumen of the vein with respect to diameter, intraluminal echogenicity and compressibility distally in the thigh to the popliteal vein.

- To be able to identify the popliteal vein in the popliteal fossa.

- To be able to assess the lumen of the popliteal vein with respect to size, intra-luminal echogenicity and compressibility distal to the point of the confluence of the anterior tibial vein, the posterior tibial vein and the fibular (peroneal) vein.

- To be able to use additional ultrasound modalities (such as colour Doppler and pulse wave Doppler) to assist in the assessment, where appropriate.

\section{Recommended number of scans}

- At least 1 year of experience at core level with a total of no less than 250 logged ePoCUS scans (of any type).

- A recommended minimum of 20 correctly performed and clinically indicated DVT ultrasound exams.

- At least $50 \%$ of the scans should demonstrate pathology.

- All scans should ideally be supervised or reviewed by an experienced and trained ePoCUS provider.

\section{Thoracic (lung) and airway ultrasound}

Thoracic and airway ePoCUS skills can be used as an adjunct in the assessment of the dyspnoeic patient in the ED. Lung ultrasound can aid in the diagnosis of pneumothorax, haemothorax, pleural effusion, pleural pathologies, lung consolidation/atelectasis and interstitial parenchymal fluid. Thoracic PoCUS can also assist with ultrasoundguided thoracocentesis. In addition, airway PoCUS can be utilised to assess intubation success in real time or after the fact.

\section{Required competencies}

- To be able to describe the indications and limitations of thoracic PoCUS.

- To be able to recognise the following key thoracic PoCUS features: - The pleural line and any abnormalities of the pleura.

- The presence or absence of lung sliding.

- The lung-pulse sign.

- A-lines.

- B-lines.

- Consolidation.

- M-mode appearance of pleura - normal and pathological.

- The lung point in pneumothorax.

- The diaphragm.

- Pleural effusions.

- The ability to integrate advanced lung PoCUS protocols into clinical practice and with specific emergency protocols (e.g. BLUE protocol and FALLS protocol).

- To be able to recognise the features of pneumonia (e.g. viral v. bacterial pneumonia).

- To be able to recognise pleural effusions and differentiate simple v. complex effusions, estimate size and to use ultrasound to guide thoracocentesis.

- To be able to differentiate cardiogenic pulmonary oedema from acute respiratory distress syndrome.

- To understand the role of lung ultrasound in guiding the management of patients on mechanical ventilation: positive endexpiratory pressure; ventilation mode; and readiness for extubation.

- The ability to recognise the following key airway PoCUS features:

- Tracheal anatomy.

- Oesophageal anatomy.
- The appearance of successful and unsuccessful tracheal intubation. Correct depth of endotracheal tube placement.

- To understand the role of airway ultrasound in guiding endotracheal intubation.

\section{Recommended number of scans}

- At least 1 year of experience at core level with a total of no less than 250 logged ePoCUS scans (of any type).

- A recommended minimum of 25 correctly performed and clinically indicated lung ultrasound exams.

- At least $50 \%$ of the scans should demonstrate pathology or require ultrasound for procedures.

- All scans should ideally be supervised or reviewed by an experienced and trained ePoCUS provider.

\section{Regional anaesthesia and nerve blocks}

Nerve blocks (peripheral nerve blocks and plexus nerve blocks) are extremely important in the ED. They can facilitate procedures such as dislocated joint reductions and provide analgesia for painful injuries. Local anaesthetic alternatives to opioids are valued highly amid the current opioid-misuse epidemic. Peripheral or plexus nerve blockade, with or without regional anaesthesia, utilising ePoCUS, has made treating various painful conditions and procedures easier and safer in the ED. ${ }^{[9]}$

\section{Required competencies}

- To be able to describe the indications and limitations of ePoCUS for regional anaesthesia.

- To be able to safely perform the following ultrasound-guided nerve blocks:

- Interscalene brachial plexus block.

- Supraclavicular brachial plexus block.

- Forearm ultrasound-guided nerve block (FUN block) of median, radial and ulnar nerves as well as blockade of the radial nerve above the elbow.

- Femoral nerve block/femoral space block/fascia iliaca block.

Popliteal sciatic nerve block.

Serratus anterior nerve block.

\section{Recommended number of scans}

- At least 1 year of experience at core level with a total of no less than 250 logged ePoCUS scans (of any type).

- A recommended minimum of 25 correctly performed and clinically indicated ultrasound-guided nerve blocks (ideally five for each technique).

- All scans should ideally be supervised or reviewed by an experienced and trained ePoCUS provider.

\section{Hepatobiliary and genito-urinary tract PoCUS}

Hepatobiliary PoCUS is used primarily for the evaluation of patients with possible cholelithiasis and/or cholecystitis, jaundiced patients and patients with suspected ascites. The principal aims are to detect gall bladder abnormities (cholelithiasis and/or cholecystitis), dilatation of the common bile duct and identify ascites. ${ }^{[3]}$

\section{Hepatobiliary PoCUS \\ Required competencies}

- To be able to describe the indications and limitations of PoCUS of the biliary tract.

- The ability to assess the gall bladder comprehensively:

- To be able to obtain long- and short-axis views of the gall bladder. 
- To be able to identify the neck, body and fundus of the gall bladder.

- To assess the gall bladder contour and identify anatomic variants (e.g. phrygian cap).

- To assess the gall bladder internal structure and contents (e.g. stones, sludge, polyps, tumours and adenomyomatosis).

- To assess the pericholecystic space.

- To assess the gall bladder wall thickness including formal measurement.

- To assess the presence or absence of a sonographic Murphy's sign.

- To assess for complications of cholecystitis such as a gangrenous gall bladder, emphysematous gall bladder, compacted gall bladder (wall-echo-shadow (WES) sign).

- The ability to assess the biliary ducts:

- To be able to obtain transverse and longitudinal views of the common bile duct.

- To be able to use colour Doppler to assist in the identification of the common bile duct.

- To be able to identify the portal triad and measure the diameter of the common bile duct.

- The ability to assess the liver:

- To be able to measure the liver and assess for hepatomegaly.

- To be able to assess for any gross parenchymal abnormalities (e.g. cysts, abscesses, masses or tumours).

- To be able to identify features of liver cirrhosis and portal hypertension.

\section{Recommended number of scans}

- At least 1 year of experience at core level with a total of no less than 250 logged ePoCUS scans (of any type).

- A recommended minimum of 20 correctly performed and clinically indicated hepatobiliary scans.

- At least $50 \%$ of the scans should demonstrate pathology.

- All scans should ideally be supervised or reviewed by an experienced and trained ePoCUS provider.

\section{Genito-urinary PoCUS}

Genito-urinary PoCUS is primarily for the evaluation of a patient with flank pain, haematuria, urinary retention and/or renal impairment. ${ }^{[3]}$

\section{Required competencies}

- To be able to describe the indications and limitations of PoCUS of the genito-urinary tract.

- To be able to ultrasonographically assess the renal tract:

- To be able to obtain long- and short-axis views of both kidneys.

- To be able to obtain measurements of size, length and width of the kidney.

- To be able to assess for hydronephrosis (none, mild, moderate, severe and complicated pyonephrosis).

- To be able to assess for dilated proximal ureters.

- To be able to detect and assess simple renal cysts, complex renal cysts and renal tumours.

- To be able to detect normal variant and abnormal renal anatomy e.g. polycystic kidneys, extrarenal pelvis, column of Bertin, dromedary hump, horseshoe kidney, duplex kidney, pelvic kidney and absent kidney.

- To be able to integrate ePoCUS findings into a treatment algorithm for the management of renal calculi.

- To be able to identify pyelonephritis and its complications.

- To be able to assess the bladder:

- To be able to obtain long- and short-axis views of the bladder.
- To be able to assess bladder volume.

To be able to detect bladder calculi if possible.

- To be able to identify any variants of the bladder wall and recognise the significance of these, e.g. diverticulae, tumours.

- To be able to appreciate the relation of the bladder to other pelvic structures in males and females.

- To assist with procedural guidance with regards to suprapubic catheter insertion.

- To assist with the identification of urethral and bladder foreign bodies.

\section{Recommended number of scans}

- At least 1 year of experience at core level with a total of no less than 250 logged ePoCUS scans (of any type).

- A recommended minimum of 20 correctly performed and clinically indicated genito-urinary scans.

- At least $50 \%$ of the scans should demonstrate pathology.

- All scans should ideally be supervised or reviewed by an experienced and trained ePoCUS provider.

\section{Gastrointestinal tract PoCUS}

This application of gastrointestinal tract PoCUS is primarily for the evaluation of the patient with suspected appendicitis, extrapulmonary tuberculosis as well as the patient with suspected bowel obstruction.

\section{Required competencies}

- To be able to describe the indications and limitations of bowel PoCUS.

- To be able to recognise and differentiate between small and large bowel.

- To be able to use ultrasound to detect:

- Intra-abdominal free fluid and for guidance of paracentesis.

- Abdominal tuberculosis in addition to other components of the FASH protocol (focused assessment using sonography for HIVassociated tuberculosis).

- Appendicitis.

- Bowel obstruction.

- Pneumoperitoneum.

Diverticulitis.

- Inguinal hernia.

- Intussusception.

Pyloric stenosis.

\section{Recommended number of scans}

- At least 1 year of experience at core level with a total of no less than 250 logged ePoCUS scans (of any type).

- A recommended minimum of 50 correctly performed and clinically indicated gastrointestinal scans.

- At least $50 \%$ of the scans should demonstrate pathology.

- All scans should ideally be supervised or reviewed by an experienced and trained ePoCUS provider.

\section{Focused obstetric and gynaecological PoCUS}

ePoCUS for the female patient can assist in the assessment of abdominal pain, vaginal bleeding, shock, pregnancy and pre-term labour. An important aspect of focused obstetric ultrasound is the mitigation of medico-legal risk. The emergency purpose of the scan as well as its limited or focused nature should be explained to the patient and family. In addition, it should be stated clearly that the scan is not intended to identify foetal abnormalities. These discussions should be clearly documented in the clinical records. In general, 
focused obstetric ultrasound will mostly be used in first-trimester pregnancies, but there will be indications for use in pregnancies that are further advanced (e.g. identification of foetal heart or risk factors for threats to the pregnancy).

Focused obstetric and gynaecological PoCUS applications include but are not limited to:

- Identification of an intra-uterine pregnancy.

- Identification of the foetal heart (using M-mode and not pulse wave Doppler) and other poor prognostic signs for a first-trimester pregnancy.

- Identification of suspicious signs of ectopic pregnancy.

- Identification of miscarriage.

- Identification of placenta praevia.

- Identification of abruptio placentae.

- Identification of ultrasound indications of pre-term labour.

- Identification of ovarian torsion.

- Identification of pelvic inflammatory disease including tuboovarian abscess.

- Identification of ovarian cysts and knowledge of criteria for referral.

- Identification of uterine fibroids.

\section{Required competencies}

Focused obstetric and gynaecological PoCUS competencies should be achieved with either transabdominal and/or transvaginal scanning:

- To be able to identify the uterus cervix, body and fundus in longitudinal and short axes.

- To be able to identify the uterine cornua and follow both fallopian tubes laterally.

- To be able to identify the pouch of Douglas and assess for free fluid in both axes.

- To be able to identify the ovaries.

- To be able to identify the internal and external iliac vessels and lymphadenopathy.

- To be able to identify adnexal cysts and masses.

- To be able to describe the indications, clinical algorithm, and limitations of PoCUS in first-trimester pregnancy pain and bleeding.

- To be able to detect an intra-uterine pregnancy, differentiate between a live foetus, foetal death and molar pregnancy.

- To be able to identify the intra-decidual sac sign, gestational sac, yolk sac and embryo.

- To be able to identify the foetal heart and quantify the heart rate using M-mode.

- To be able to estimate gestational age (crown-rump length/ bi-parietal diameter/femur length).

- To be able to identify an ectopic pregnancy.

- To be able to measure the endometrial stripe thickness.

- To be able to identify abruptio placentae and placenta praevia.

\section{Recommended number of scans}

- At least 1 year of experience at core level with a total of no less than 250 logged ePoCUS scans (of any type).

- A recommended minimum of 40 correctly performed and clinically indicated focused obstetric and gynaecological PoCUS scans.

- At least $50 \%$ of the scans should demonstrate pathology.

- All scans should ideally be supervised or reviewed by an experienced and trained ePoCUS provider.

\section{Transcranial Doppler and ocular ultrasound}

Transcranial Doppler can give information regarding cerebral blood-flow velocities. This information can be used to diagnose cerebrovascular spasm as well as cerebrovascular flow abnormalities from critically elevated intracranial pressure. ${ }^{[10]}$

\section{Required competencies}

- To be able to describe the indications and limitations of transcranial Doppler PoCUS.

- To be able to identify the relevant cerebral vessel ultrasound anatomy.

- To be able to evaluate both anterior and posterior circulations.

- To be able to identify cerebral vasospasm.

- To be able to identify the presence of cerebral midline shift.

- To be able to identify brain death from cessation of blood flow.

- Ocular ePoCUS can assist in the assessment of patients with decreased vision/loss of vision, suspected foreign body, ocular pain and eye trauma. It can also provide information in the setting of patients with suspected raised intracranial pressure. ${ }^{[1]]}$

\section{Required competencies}

- To be able to describe the indications and limitations of ocular PoCUS.

- To be able to identify relevant ultrasound anatomy of the globe and orbital structures.

- To be able to identify extra-ocular movement and pupillary reactivity.

- To be able to identify papilloedema.

- To be able to perform PoCUS protocols for the detection of vitreous haemorrhage, retinal detachment, lens dislocation, retrobulbar haematoma, foreign body and other pathologies.

- To be able to perform optic nerve sheath diameter measurements to assess for raised intracranial pressure.

\section{Recommended number of scans}

- At least 1 year of experience at core level with a total of no less than 250 logged ePoCUS scans (of any type).

- A recommended minimum of 40 correctly performed, clinically indicated transcranial Doppler and ocular scans.

- At least $50 \%$ of the scans should demonstrate pathology.

- All scans should ideally be supervised or reviewed by an experienced, trained ePoCUS provider.

1. Bruijns SR, Engelbrecht D, Lubinga W, Wells M, Wallis LA. Penetrating the acoustic shadows Emergency ultrasound in South African emergency departments. S Afr Med J 2008;98(12):932-934.

2. Wells M, Bruijns S. College of Emergency Medicine of South Africa policy document. Emergency Wells M, Bruijns S. College of Emergency Medicine of South Africa policy document. Emergency
Ultrasound in South Africa. Johannesburg: EMSSA, 2009. https://emssa.org.za/documents/CEM(SA)Ultrasound in South Africa. Johannesburg: EMSSA, 2009. https://
Emergency-Ultrasound-POLICY.pdf (accessed I October 2020).

3. United Kingdom Royal College of Emergency Medicine (RCEM). Guidance for level 2 ultrasound . United Kingdom Royal College of Emergency Medicine (RCEM). Guidance for level 2 ultrasound
practice in emergency medicine. London: RCEM, 2008. https://www.rcem.ac.uk/docs/ practice in emergency medicine. London: RCEM, 2008. https://www.rcem
Training/1.14.6\%20RCEM_Level_2_Guidance-1\%20(1).pdf (accessed 1 October 2020).

4. American College of Emergency Physicians (ACEP). Ultrasound guidelines: Emergency, pointof-care, and clinical ultrasound guidelines in medicine. Irving: ACEP, 2016. https://www.acep org/patient-care/policy-statements/ultrasound-guidelines-emergency-point-of-care-and-clinicalultrasound-guidelines-in-medicine/ (accessed 1 October 2020).

5. Australasian College for Emergency Medicine (ACEM). Policy on the use of focused ultrasound in emergency medicine. Melbourne: ACEM, 2016. https://acem.org.au/getmedia/0702004f-c669-4646b5fc-4fa577117ba3/COR742_vl_ED_Ultrasound_Training_Governance_Guideline (accessed 1 October 2020).

6. International Federation for Emergency Medicine (IFEM). Emergency ultrasound special interest group Point-f are ultaso cc/wp-content/uploads/2016/07/IFEM-Point-of-Care-Ultrasound-Curriculum-Guidelines-2014.pdf cc/wp-content/uploads/2016/
(accessed 1 October 2020)

7. Canadian Association of Emergency Physicians (CAEP). The CAEP emergency ultrasound curriculum - objectives and recommendations for implementation in postgraduate training. Ottawa: CAEP, 2016. https://caep.ca/wp-content/uploads/2018/09/EUC-position-statement-English-full.pdf (accessed October 2020)

8. Mosier JM, Martin J, Andrus P, et al. Advanced hemodynamic and cardiopulmonary ultrasoun for critically ill patients in the emergency department. Emerg Med 2018;50(1):17-34. https://doi. org $/ 10.12788 /$ emed.2018.0078

9. Herring, AA. Bringing ultrasound-guided regional anaesthesia to emergency medicine. AEM Edu Training 2017;1:165-168. https://doi.org/10.1002/aet2.10027

10. Blanco P, Blaivas M. Applications of transcranial color-coded sonography in the emergency department. J Ultrasound Med 2017;36:1251-1266 https://doi.org/10.7863/ultra.16.04050

1. Adhikari SR. Ocular ultrasound. Sonoguide ultrasound guide for emergency physicians. Irving: ACEP. https://www.acep.org/sonoguide/smparts_ocular.html (accessed 1 October 2020). 


\section{Part 5: Quality management in emergency point-of- care ultrasound}

Whether in a university programme or in a single ED, EMSSA recommends that where ePoCUS is performed, it must be governed by a quality management programme. This programme should include requirements for leadership, supervision of training, record-keeping, quality management and matters relating to ultrasound machines. A written policy should be in place to address each of these issues, iterated below (good examples can be found on the webpages of the ACEP and the Canadian Association of Emergency Physicians). ${ }^{[1,2]}$

\section{Leadership}

All hospitals with an ED where PoCUS is performed should have a designated ultrasound clinician lead. The ultrasound lead will be responsible for coordinating administration, education, quality management and research.

\section{Supervision of training}

Most competencies in PoCUS are learned in the work environment while performing clinically indicated scans under the guidance of an experienced supervisor. It is therefore essential that a programme be in place for the supervision and education of junior PoCUS users in the ED. This is necessary for both enhanced learning as well as preparation for the credentialing process.

\section{Record-keeping}

Every scan performed in the ED must be supported by appropriate records. This documentation should include relevant still or video ultrasound images as well as a written description and interpretation of the findings in the clinical records. How the images are archived and retained should be determined by the needs and resources of each department.

\section{Quality management}

Quality management is a broad concept that encompasses other aspects mentioned in this section, such as image archiving and documentation, but also deals with quality assurance and quality improvement. In this context, quality assurance is a process of periodic auditing of the compliance of users with the departmental policies and protocols as well as auditing of the ultrasound skills of the users within the department. Based on the findings of the audit, directed quality improvement programmes can be instituted to address any deficiencies identified. Every department should have a policy on this process of quality management.

\section{Ultrasound machines}

Policies should be established to govern the characteristics of machines that are used in the department (e.g. portability, size, functionality, available modes and imaging capability, boot-up time and archiving options), as well as other important issues such as infection control and safe use of the machine.

1. Ultrasound guidelines: Emergency, point-of-care and clinical ultrasound guidelines in medicine.
Ann Emerg Med 2017;69(5):e27-e54. https://doi.org/10.1016/j.annemergmed.2016.08.457
2. Lewis D, Rang L, Kim D, et al. Recommendations for the use of point-of-care ultrasound (POCUS)
by emergency physicians in Canada. CJEM 2019;21(6):721-726. https://doi.org/10.1017/ cem.2019.392 